\title{
ON DISK-LIKE SELF-AFFINE TILES ARISING FROM POLYOMINOES*
}

\author{
JOHANNES GMAINER ${ }^{\dagger}$ AND JÖRG M. THUSWALDNER ${ }^{\ddagger}$
}

\begin{abstract}
In this paper we study a class of plane self-affine lattice tiles that are defined using polyominoes. In particular, we characterize which of these tiles are homeomorphic to a closed disk. It turns out that their topological structure depends very sensitively on their defining parameters.

In order to achieve our results we use an algorithm of Scheicher and the second author which allows to determine neighbors of tiles in a systematic way as well as a criterion of Bandt and Wang, with that we can check disk-likeness of a self-affine tile by analyzing the set of its neighbors.
\end{abstract}

Key words. polyomino, self-affine tile, topological disk

AMS subject classifications. 28A80, 05B50, 54F65

1. Introduction. Let $A$ be an $m \times m$ integer matrix that is expanding, i.e., each of its eigenvalues is greater than 1 in modulus. Suppose that $|\operatorname{det}(A)|>1$ and let $\mathcal{D} \subset \mathbb{Z}^{m}$ be a complete set of coset representatives of $\mathbb{Z}^{m} / A \mathbb{Z}^{m}$. By a result of Hutchinson [10] there exists a unique non-empty compact set $\mathcal{T}:=\mathcal{T}(A, \mathcal{D})$ such that

$$
A \mathcal{T}=\bigcup_{d \in \mathcal{D}}(\mathcal{T}+d)
$$

The set $\mathcal{T}$ is called an integral self-affine tile with standard digit set. (cf. Lagarias and Wang [14]). In [15] it is shown that for an integral self-affine tile $\mathcal{T}$ with standard digit set there always exists a set $L$ of translations such that $\mathcal{T}+L$ forms a tiling of $\mathbb{R}^{m}$. The tiles $\mathcal{T}=\mathcal{T}(A, \mathcal{D})$ discussed in the present paper are integral self-affine tiles with standard digit set that tile by the lattice $\mathbb{Z}^{m}$, i.e.,

$$
\mathcal{T}+\mathbb{Z}^{m}=\mathbb{R}^{m}
$$

where

$$
\mu_{m}\left(\left(\mathcal{T}+\gamma_{1}\right) \cap\left(\mathcal{T}+\gamma_{2}\right)\right)=0 \quad \text { for } \quad \gamma_{1} \neq \gamma_{2} \quad\left(\gamma_{1}, \gamma_{2} \in \mathbb{Z}^{m}\right) .
$$

Here $\mu_{m}$ denotes the $m$-dimensional Lebesgue measure. Following Bandt and Wang [4] we refer to such a tile as $\mathbb{Z}^{m}$-tile for short. There are standard methods for checking whether $\mathcal{T}$ forms a tiling or not. We refer for instance to Vince [24, Theorem 4.2] where a list of tiling criteria is given.

This paper is devoted to the study of topological properties of a class of $\mathbb{Z}^{2}$-tiles. Let us first give a short review of previous results on topological properties of selfaffine tiles and attractors of iterated function systems (IFS) in general. Hata [8, 9] discusses basic topological properties like connectivity and local connectivity of IFS. The problem of connectivity of IFS is also treated by Kirat and Lau [12] as well as Kirat et al. [13]. Especially, plane IFS were studied thoroughly. Luo et al. [16] deal

\footnotetext{
*Received March 10, 2005; accepted for publication March 16, 2007.

†Institut für Diskrete Mathematik und Geometrie, Technische Universität Wien, Wiedner Hauptstraße 8-10, A-1040 Wien, Austria (jgmainer@osiris.tuwien.ac.at).

${ }^{\ddagger}$ Institut für Mathematik und Angewandte Geometrie, Abteilung für Mathematik und Statistik, Montanuniversität Leoben, Franz-Josef-Straße 18, A-8700 Leoben, Austria (Joerg.Thuswaldner @unileoben.ac.at). The second author was supported by the FWF project P17557-N12.
} 
with plane IFS that are homeomorphic to a closed disk (disk-like) while Ngai and Tang $[18,19,20]$ study properties of plane IFS having a more difficult topological structure. Bandt and Wang [4] gave a criterion for the disk-likeness of a $\mathbb{Z}^{2}$-tile $\mathcal{T}$ in terms of its "neighbors". Finding the neighbors of a $\mathbb{Z}^{2}$-tile was discussed in Strichartz and Wang [23] as well as in Scheicher and Thuswaldner [21]. Classes of $\mathbb{Z}^{2}$-tiles related to number systems are studied by Akiyama et al. in [1,2]. For a survey on results related to the topology of IFS we refer to Akiyama and Thuswaldner [3].

In the present paper we want to investigate topological properties of a class of $\mathbb{Z}^{2}$-tiles which are defined in terms of polyominoes (cf. Golomb [6, Chapter 1] for the definition of a polyomino). We will call such tiles polyomino typed tiles. Polyomino typed tiles were first introduced by Song and Kang [22]. They study basic properties of these tiles. Furthermore, they give - without stating an exact proof - some examples for polyomino typed tiles that are homeomorphic to a closed disk. This forms the starting point of the present paper. Our goal will be to give a large class of polyomino typed tiles that are homeomorphic to a closed disk.

Following Song and Kang [22] we will now describe how polyomino typed tiles are defined.

Let us assume in the sequel that a polyomino $P$ is embedded into $\mathbb{R}^{2}$ such that it can be regarded as a finite union of Voronoi cells

$$
V(x)=\left\{y \in \mathbb{R}^{2}|| y-x|\leq| y-z \mid \text { for all } z \in \mathbb{Z}^{2}\right\} \quad\left(x \in \mathbb{Z}^{2}\right) .
$$

This means that there exists a set $\mathcal{D}(P) \subset \mathbb{Z}^{2}$ such that

$$
P=\bigcup_{x \in \mathcal{D}(P)} V(x) .
$$

We call $P$ exact if it tiles the plane in the sense that there exists a lattice $\Gamma$ generated by $l_{1}, l_{2} \in \mathbb{Z}^{2}$ such that

$$
P+\Gamma=\mathbb{R}^{2} \quad \text { and } \quad \mu_{2}(P \cap(P+\gamma))=0 \quad \text { for each } \gamma \in \Gamma \backslash\{0\}
$$

(cf. [5]). If $P$ is exact, set

$$
\Sigma:=\{s \in \Gamma \mid P \cap(P+s) \neq \emptyset\} .
$$

Let $e_{1}, e_{2}$ denote the canonical base vectors of $\mathbb{R}^{2}$. Then we call $A$ a surrounding matrix of the polyomino $P$ if $A$ is regular and if there exist $s_{1}, s_{2} \in \Sigma$ such that

$$
A e_{1}=s_{1} \quad \text { and } \quad A e_{2}=s_{2} .
$$

Of course there exist several surrounding matrices for a given exact polyomino $P$.

With these definitions it is easy to see that the following lemma holds (cf. also $[22])$.

Lemma 1.1. If $P$ is an exact polyomino and $A$ is one of its expanding surrounding matrices then $\mathcal{T}(A, \mathcal{D}(P))$ is an integral self-affine tile with standard digit set.

As mentioned before, a tile $\mathcal{T}$ which is defined by an exact polyomino as in Lemma 1.1 is called a polyomino typed tile. First we note that it seems to be a very difficult problem to characterize the sets $\mathcal{D}(P)$ for which $P$ is an exact polyomino. However, in [5] one can find an easy criterion which allows to decide whether a given 
polyomino is exact or not (see Lemma 3.1 below). More generally, there is an algorithm by Keating and Vince [11] which can decide for a given polyomino if it admits an isohedral tiling or not. Furthermore, exact polyominoes can be characterized by some easy properties (cf. [5]). We will deal with a subclass of polyominoes for which the exactness can be shown immediately.

Definition 1.2. Let $P$ be a polyomino. We call $P$ stair-like if the set $\mathcal{D}(P)$ can be written as

$$
\mathcal{D}=\mathcal{D}(P)=\left\{d_{1}, \ldots, d_{q} \mid d_{i} \in \mathbb{Z}^{2} \text { and }\left\{d_{2}-d_{1}, \ldots, d_{q}-d_{q-1}\right\} \subseteq\{(1,0),(0,1)\}\right\} .
$$
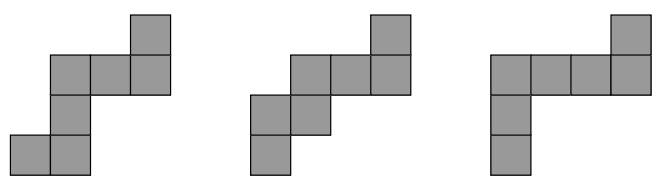

FIG. 1. Three examples of stair-like polyominoes

We call these polyominoes stair-like because of their shape. Figure 1 contains three examples of stair-like polyominoes. Accordingly, the $\mathbb{Z}^{2}$-tile emerging from a stair-like polyomino will be called stair-like tile. It is worth to note that the stair-like structure of $P$ does in general not reflect to the corresponding tile $\mathcal{T}$. Figures 11, 12 and 13 contain the tiles (and their neighbors) that arise from the polyominoes of Figure 1.

The aim of this paper is to characterize stair-like tiles which are disk-like. To this matter we will combine three results.

- First we will use a criterion of Gröchenig and Haas [7] that permits us to prove that stair-like tiles are $\mathbb{Z}^{2}$-tiles. In fact, to this matter it is sufficient to prove that they tile $\mathbb{R}^{2}$ by $\mathbb{Z}^{2}$-translates.

- In a second step we determine the "neighbors" of these tiles. This can be done in a systematic way by using the neighbor finding algorithm discussed in Scheicher and Thuswaldner [21]. ${ }^{1}$

- Then we apply the criterion contained in Bandt and Wang [4] which allows to decide whether a $\mathbb{Z}^{2}$-tile is disk-like or not by inspecting its neighbors.

The paper is organized as follows. In the next section we discuss the neighbor finding algorithm and the criterion for disk-likeness. Section 3 is devoted to some properties of polyominoes. The main result of the present paper is contained in Section 4 (Theorem 4.2; see also Remark 4.4). Section 5 contains the proof of this result. The paper ends with some examples.

2. Preliminaries. In what follows let $\mathcal{T}=\mathcal{T}(A, \mathcal{D})$ be an integral self-affine tile with standard digit set. This section is devoted to the presentation of the tools needed in the proof of our main result. First we define a certain graph that will be needed throughout the proof. Then we describe the criterion of Gröchenig and Haas [7] that will permit us to conclude that stair-like tiles are $\mathbb{Z}^{2}$-tiles. After that an algorithm of Scheicher and Thuswaldner [21] for finding the neighbors of a $\mathbb{Z}^{m}$-tile is presented.

\footnotetext{
${ }^{1}$ Note that the neighbor finding algorithm in Strichartz and Wang [23] can not be applied in our setting as the occurring matrices can have eigenvalues arbitrarily close to 1 .
} 
This algorithm is needed to apply a criterion of Bandt and Wang [4] which will finally permit us to decide whether a given $\mathbb{Z}^{2}$-tile is homeomorphic to a closed disk or not.

Define the graph $G\left(\mathbb{Z}^{m}\right)$ with set of states $V=\mathbb{Z}^{m}$ in the following way. There exists an edge $r \stackrel{d \mid d^{\prime}}{\longrightarrow} r^{\prime}$ from $r$ to $r^{\prime}$ labelled by $d \mid d^{\prime}$ if $A r+d^{\prime}=r^{\prime}+d$ holds for a pair $\left(d, d^{\prime}\right) \in \mathcal{D} \times \mathcal{D}$. In what follows, we will write $E\left(G\left(\mathbb{Z}^{m}\right)\right)$ for the set of edges of $G\left(\mathbb{Z}^{m}\right)$.

For each subset $M \subset \mathbb{Z}^{m}$ we define the graph $G(M)=G_{A, \mathcal{D}}(M)=(V, E)$ to be the restriction of $G\left(\mathbb{Z}^{m}\right)$ to the set of vertices $M$.

Set $R_{0}:=\left\{0, \pm e_{1}, \pm e_{2}\right\}$ and define $R_{n}$ inductively by

$$
R_{n}:=R_{n-1} \cup\left\{k \in \mathbb{Z}^{m} \mid(A k+\mathcal{D}) \cap(l+\mathcal{D}) \neq \emptyset \quad \text { for } \quad l \in R_{n-1}\right\} .
$$

Define the set

$$
R=R(A, \mathcal{D})=\bigcup_{n \geq 0} R_{n}
$$

$R$ has to be a finite set. In particular we have $R_{n-1}=R_{n}$ for $n$ large enough (cf. Gröchenig and Haas [7, Section 4]). Indeed, it is easy to see from the fact that $A$ is expanding that the increasing sequence $\left(R_{n}\right)_{n \geq 0}$ has to be bounded. $R$ as well as $G(R)$ can be constructed easily by the above definition.

Remark 2.1. It is clear that $G_{A, \mathcal{D}}(M)$ and $R(A, \mathcal{D})$ depend on the defining parameters $A$ and $\mathcal{D}$ of the associated $\mathbb{Z}^{m}$-tile $\mathcal{T}(A, \mathcal{D})$. However, most of the time it is clear from the context to which tile these objects refer. In these cases we will just write $G(M)$ and $R$ in order to avoid tedious notation.

The following criterion due to Gröchenig and Haas [7, Section 4] will be needed later.

Lemma 2.2. Let $\mathcal{T}=\mathcal{T}(A, \mathcal{D})$ be an integral self-affine tile with standard digit set. If the spectral radius of the accompanying matrix of $G(R \backslash\{0\})$ is less than $|\operatorname{det}(A)|$ then $\mathcal{T}$ is a $\mathbb{Z}^{m}$-tile.

Recall that $s \in \mathbb{Z}^{m} \backslash\{0\}$ is a neighbor of a $\mathbb{Z}^{m}$-tile $\mathcal{T}$ if $\mathcal{T} \cap(\mathcal{T}+s) \neq \emptyset$ (cf. Scheicher and Thuswaldner [21] $)^{2}$. A neighbor $s$ is a vertex neighbor if $\mathcal{T} \cap(\mathcal{T}+s)$ contains only one point. If $\mathcal{T} \cap(\mathcal{T}+s)$ contains uncountably many points, then $s$ is called edge neighbor. Our first task will be the determination of the set

$$
S=S(A, \mathcal{D}):=\left\{s \in \mathbb{Z}^{m} \backslash\{0\} \mid \mathcal{T} \cap(\mathcal{T}+s) \neq \emptyset\right\}
$$

of neighbors of a given $\mathbb{Z}^{m}$-tile $\mathcal{T}$. The restriction $G(S)$ of $G\left(\mathbb{Z}^{m}\right)$ to the set of neighbors $S$ is called the neighbor graph of $\mathcal{T}$.

Certain properties of $G(S)$ have been proved (cf. for instance [17, 21]). We will use the fact that $G(S \cup\{0\})$ is the union of all cycles and paths connecting two cycles in $G\left(\mathbb{Z}^{m}\right)$ (cf. [21]). The graph $G(S)$ also contains information on the sets $\mathcal{T} \cap(\mathcal{T}+s)$ $(s \in S)$. Indeed, we have

$$
\begin{aligned}
& \mathcal{T} \cap(\mathcal{T}+s)= \\
& \quad\left\{\sum_{i \geq 1} A^{-i} d_{i}\left|d_{1}\right| d_{1}^{\prime}, d_{2} \mid d_{2}^{\prime} \ldots \text { is the labelling of a walk in } G(S) \text { starting at } s\right\} .
\end{aligned}
$$

\footnotetext{
${ }^{2}$ In what follows we will use the term "neighbor" ambigously for the point $s$ and for the set $\mathcal{T}+s$. This will cause no confusion.
} 
Scheicher and Thuswaldner [21] found that $G(R)$ is a subgraph of $G(S \cup\{0\})$. Moreover, they found a way to construct $G(S)$ starting from $G(R)$ by using a certain graph product. We will now define this graph product and present the construction method. To this matter we need the following reduction operation. Let $G$ be a directed graph. We denote by $\operatorname{Red}(G)$ the graph that emerges from $G$ if all states of $G$, which are not starting points of a walk of infinite length, are removed.

Definition 2.3. Let $\mathcal{T}(A, \mathcal{D})$ be a $\mathbb{Z}^{m}$-tile and let $G_{1}$ and $G_{1}^{\prime}$ be subgraphs of $G_{A, \mathcal{D}}\left(\mathbb{Z}^{m}\right)$. The product $G_{2}:=G_{1} \otimes G_{1}^{\prime}$ is defined in the following way. Let $r_{1}, s_{1}$ be states of $G_{1}$ and $r_{1}^{\prime}, s_{1}^{\prime}$ be states of $G_{1}^{\prime}$. Furthermore, let $\ell_{1}, \ell_{1}^{\prime}, \ell_{2} \in \mathcal{D}$.

- $r_{2}$ is a state of $G_{2}$ if $r_{2}=r_{1}+r_{1}^{\prime}$.

- There exists an edge $r_{2} \stackrel{\ell_{1} \mid \ell_{2}}{\longrightarrow} s_{2}$ in $G_{2}$ if there exist

$$
r_{1} \stackrel{\ell_{1} \mid \ell_{1}^{\prime}}{\longrightarrow} s_{1} \in E\left(G_{1}\right) \quad \text { and } \quad r_{1}^{\prime} \stackrel{\ell_{1}^{\prime} \mid \ell_{2}}{\longrightarrow} s_{1}^{\prime} \in E\left(G_{1}^{\prime}\right)
$$

with $r_{1}+r_{1}^{\prime}=r_{2}$ and $s_{1}+s_{1}^{\prime}=s_{2}$ or there exist

$$
r_{1} \stackrel{\ell_{1}^{\prime} \mid \ell_{2}}{\longrightarrow} s_{1} \in E\left(G_{1}\right) \quad \text { and } \quad r_{1}^{\prime} \stackrel{\ell_{1} \mid \ell_{1}^{\prime}}{\longrightarrow} s_{1}^{\prime} \in E\left(G_{1}^{\prime}\right)
$$

with $r_{1}+r_{1}^{\prime}=r_{2}$ and $s_{1}+s_{1}^{\prime}=s_{2}$.

It is easily seen that a graph $G$ containing 0 in its set of vertices is isomorphic to a subgraph of $G \otimes G$ :

Suppose that $r_{1} \stackrel{\ell_{1} \mid \ell_{1}^{\prime}}{\longrightarrow} s_{1} \in E(G)$, then also $0 \stackrel{\ell_{1}^{\prime} \mid \ell_{1}^{\prime}}{\longrightarrow} 0 \in E(G)$. The second part of Definition 2.3 tells us that $r_{1}+0 \stackrel{\ell_{1} \mid \ell_{1}^{\prime}}{\longrightarrow} s_{1}+0 \in E(G \otimes G)$.

Let $\mathcal{T}(A, \mathcal{D})$ be a $\mathbb{Z}^{m}$-tile. The graph $G(S)$, and with it the set $S$ of neighbors, can be determined by the following algorithm starting from the graph $G(R)$ (cf. [21]).

Algorithm 2.4.

$$
\begin{aligned}
& p:=1 \\
& B[1]:=\operatorname{Red}(G(R)) \\
& \text { repeat } \\
& \quad p:=p+1 \\
& \quad B[p]:=\operatorname{Red}(B[p-1] \otimes B[1]) \\
& \text { until } B[p]=B[p-1]
\end{aligned}
$$

It is proved that this algorithm always terminates after finitely many, say $p_{0}$, steps and $G(S)=B\left[p_{0}\right] \backslash\{0\}$.

Often the graph $G(R)$ is of a very simple structure so that it can be determined for whole classes of tiles at once. Moreover, in the case of stair-like tiles Algorithm 2.4 terminates very soon so that it gives us the graph $G(S)$ very quickly.

The following lemma yields a non-trivial and somewhat unexpected result. Despite the tiles $\mathcal{T}(A, \mathcal{D})$ and $\mathcal{T}(-A, D)$ are of different shape, their neighbor sets coincide.

Lemma 2.5. Given a matrix $A \in \mathbb{Z}^{m \times m}$ and $\mathcal{D} \subset \mathbb{Z}^{m}$ with $|\mathcal{D}|=|\operatorname{det} A| a$ complete set of coset representatives for $\mathbb{Z}^{m} / A \mathbb{Z}^{m}$. Then

$$
S(A, \mathcal{D})=S(-A, \mathcal{D})
$$


i.e., $\mathcal{T}_{1}=\mathcal{T}_{1}(A, \mathcal{D})$ and $\mathcal{T}_{2}=\mathcal{T}_{2}(-A, \mathcal{D})$ have the same neighbors.

Proof. In the graph $G_{A, \mathcal{D}}(S \cup\{0\})$ there exists by definition a labelled edge $s_{1} \stackrel{d_{2} \mid d_{1}}{\longrightarrow} s_{2}$, if $A s_{1}=s_{2}+\left(d_{2}-d_{1}\right)$ holds. So $s_{1} \stackrel{d_{1} \mid d_{2}}{\longrightarrow}-s_{2}$ exists in the graph $G_{-A, \mathcal{D}}(S \cup\{0\})$. Now we have to think over what happens with cycles and the paths connecting two cycles if we substitute $A$ by $-A$ :

Given a state $s=s_{1}$ in a cycle $s=s_{1} \rightarrow s_{2} \cdots \rightarrow s_{M}=s_{1}$ in $G_{A, \mathcal{D}}(S \cup\{0\})$, we easily see that $s$ is also contained in the graph $G_{-A, \mathcal{D}}(S \cup\{0\})$ : As there also exists the cycle $-s_{1} \rightarrow-s_{2} \cdots \rightarrow-s_{M}=-s_{1}$ in $G_{A, \mathcal{D}}(S \cup\{0\})$, the state $s$ either belongs to the cycle $s=s_{1} \rightarrow-s_{2} \rightarrow s_{3} \cdots \rightarrow s_{M}=s_{1}$ if $M$ is odd, or to the cycle $s=s_{1} \rightarrow-s_{2} \rightarrow s_{3} \cdots \rightarrow s_{M-1} \rightarrow-s_{1} \rightarrow s_{2} \cdots \rightarrow s_{1}=s$ if $M$ is even. This is why $s \in G_{-A, \mathcal{D}}(S \cup\{0\})$. In an analogous manner, the states on paths connecting two cycles are again on paths connecting two cycles when changing from $A$ to $-A$. Finally, after changing the roles of $A$ and $-A$ we are done.

Let $\mathcal{T}=\mathcal{T}(A, \mathcal{D})$ be a $\mathbb{Z}^{m}$-tile. We also mention the following symmetry property of $G(S \cup\{0\})$. If $s \stackrel{d \mid d^{\prime}}{\longrightarrow} s^{\prime} \in E(G(S))$ then $-s \stackrel{d^{\prime} \mid d}{\longrightarrow}-s^{\prime} \in E(G(S))$. Thus in order to show that $\pm s \in S$ it suffices to show that $s \in S$. The same is true for the graphs $G(R)$ and $B[p](p \geq 1)$.

We want to know if $\mathcal{T}=\mathcal{T}(A, \mathcal{D}(P))$ is disk-like. As the following fundamental results of Bandt and Wang [4] show, the answer to this question depends on the number of neighbors of $\mathcal{T}$, i.e., $|S|$, and the connectivity of $\mathcal{D}(P)$ :

Proposition 2.6. Let $\mathcal{T}$ be a disk-like $\mathbb{Z}^{2}$-tile. Then in the tiling $\mathcal{T}+\mathbb{Z}^{2}$ one of the following must be true.

1. $\mathcal{T}$ has no vertex neighbors and 6 edge neighbors $\pm \alpha, \pm \beta, \pm(\alpha+\beta)$ for some $\alpha, \beta \in \mathbb{Z}^{2}$, and $\mathbb{Z} \alpha+\mathbb{Z} \beta=\mathbb{Z}^{2}$.

2. $\mathcal{T}$ has 4 edge neighbors $\pm \alpha, \pm \beta$ and 4 vertex neighbors $\pm \alpha \pm \beta$ for some $\alpha, \beta \in \mathbb{Z}^{2}$, and $\mathbb{Z} \alpha+\mathbb{Z} \beta=\mathbb{Z}^{2}$.

Definition 2.7. Let $\mathcal{F}$ be a finite subset of $\mathbb{Z}^{m}$. A subset $\mathcal{E} \subset \mathbb{Z}^{m}$ is $\mathcal{F}$-connected if for any $u, v \in \mathcal{E}$ there exists $u_{0}=u, u_{1}, \ldots, u_{n}=v \in \mathcal{E}$ with $u_{i+1}-u_{i} \in \mathcal{F}$.

Lemma 2.8. Let $\mathcal{T}(A, \mathcal{D})$ be a self-affine $\mathbb{Z}^{2}$-tile. Suppose that $\mathcal{T}$ has not more than 6 neighbors, i.e., $|S| \leq 6$. Then $\mathcal{T}$ is disk-like if and only if $\mathcal{D}$ is $S$-connected.

Lemma 2.9. Let $\mathcal{T}(A, \mathcal{D})$ be a self-affine $\mathbb{Z}^{2}$-tile. Suppose that $\mathcal{T}$ has 8 neighbors and $S=\{ \pm \alpha, \pm \beta, \pm(\alpha+\beta), \pm(\alpha-\beta)\}$. Then $\mathcal{T}$ is disk-like if and only if $\mathcal{D}$ is $\{ \pm \alpha, \pm \beta\}$-connected.

3. The surroundings of stair-like polyominoes. For the sake of completeness, in the present section we want to state some more or less obvious results on the surroundings of stair-like polyominoes.

Let the boundary $b(P)$ of a polyomino $P$ (which is a simple closed curve) be clockwise oriented and $X$ the alphabet $\{l, r, u, d\}$ (left, right, up, down). Denote by $X^{*}$ the set of finite words over $X$. For every pair $(A, B)$ of points of $b(P)$ with integer coordinates, there is a word $w \in X^{*}$, which describes the (clockwise) walk $w=(A B)$ from $A$ to $B$. In Figure 2 we have $(A B)=u r d r d r$ and $(A A)=u r d r d r d l l l u u$.

The involution $T$ of a word $w \in X^{*}$ is recursively defined as follows:

$$
T(u)=d, \quad T(d)=u, \quad T(r)=l, \quad T(l)=r,
$$




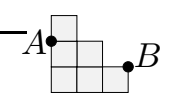

Fig. 2. A clockwise walk from $A$ to $B$

and

$$
T(w x)=T(x) T(w) \quad \forall w \in X^{*}, \quad \forall x \in X .
$$

A shorthand for $T(w)$ is $w^{\sim}$. Now we are able to state the following result of Beauquier and Nivat [5] which describes all possible surroundings of an exact polyomino in a simple way.

Lemma 3.1. (cf. [5]) A polyomino $P$ is exact if and only if there exists a point $A$ of $b(P)$ such that $(A A)$ can be written in the form $w_{1} w_{2} w_{3} w_{1}^{\sim} w_{2}^{\sim} w_{3}^{\tilde{3}}$.

After these preparations we can show that every stair-like polyomino is exact.

Proposition 3.2. For a stair-like polyomino $P$ there always exists a tiling of $\mathbb{R}^{2}$ by $\mathbb{Z}^{2}$-translates. Indeed, we can find a surrounding $w_{1} w_{2} w_{3} w_{1}^{\sim} w_{2}^{\sim} w_{3}^{\sim}$ of $P$ with

$$
\begin{array}{ll}
w_{1}=u, & w_{1}^{\sim}=d, \\
w_{2}=w \in X^{*}, & w_{2}^{\sim}=w^{\sim}, \\
w_{3}=r, & w_{3}^{\sim}=l .
\end{array}
$$

The proof of this assertion is obvious.

The six paths $w_{1}, w_{2}, w_{3}, w_{1}^{\tilde{1}}, w_{2}^{\tilde{2}}, w_{3}^{\tilde{3}}$ are exactly the segments, where the polyomino $P$ meets its six neighbors (cf. [5]).

In Figure 3 we show a tile together with a surrounding as defined in Proposition 3.2. Here $w_{1}=(A B), w_{2}=(B C), w_{3}=\left(C A^{\prime}\right), w_{1}^{\sim}=\left(A^{\prime} B^{\prime}\right), w_{2}^{\sim}=$ $\left(B^{\prime} C^{\prime}\right), w_{3}^{\tilde{3}}=\left(C^{\prime} A\right)$.

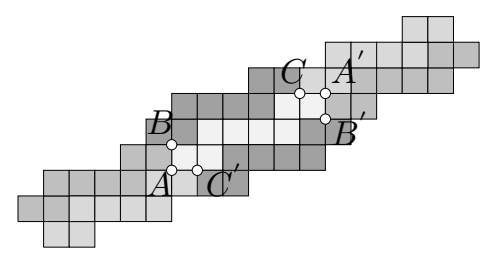

FIG. 3. This surrounding always exists

We call a stair-like polyomino irreducible if the surrounding indicated in Proposition 3.2 is the only possible surrounding for $P$. It seems to be a difficult problem to characterize all stair-like polyominoes which are irreducible.

4. Main result. Given a stair-like polyomino $P$ with $\mathcal{D}=\mathcal{D}(P)=$ $\left\{\left(\begin{array}{l}0 \\ 0\end{array}\right), \ldots,\left(\begin{array}{l}a-1 \\ b-1\end{array}\right)\right\}$, we can tile the plane by lattice translates of $P$ according to Proposition 3.2 and the remark after it. We have

$$
\mathbb{R}^{2}=\bigcup_{m, n \in \mathbb{Z}}\left(P+m l_{1}+n l_{2}\right),
$$


where $\left\{l_{1}, l_{2}\right\}$ is any ordered pair of linearly independent vectors

$$
l_{1}, l_{2} \in\left\{ \pm\left(\begin{array}{c}
1 \\
-1
\end{array}\right), \pm\left(\begin{array}{c}
a \\
b-1
\end{array}\right), \pm\left(\begin{array}{c}
a-1 \\
b
\end{array}\right)\right\} .
$$

Thus, we have 24 possibilities to put such an ordered pair into a surrounding matrix $A_{i}$ of $P$ :

$$
\begin{aligned}
& A_{1}=\left(\begin{array}{cc}
1 & a \\
-1 & b-1
\end{array}\right), \quad A_{2}=\left(\begin{array}{cc}
1 & -a \\
-1 & -b+1
\end{array}\right), \quad A_{3}=\left(\begin{array}{cc}
1 & a-1 \\
-1 & b
\end{array}\right), \\
& A_{4}=\left(\begin{array}{cc}
1 & -a+1 \\
-1 & -b
\end{array}\right), \quad A_{5}=\left(\begin{array}{cc}
a-1 & -1 \\
b & 1
\end{array}\right), \quad A_{6}=\left(\begin{array}{cc}
-a+1 & -1 \\
-b & 1
\end{array}\right) \\
& A_{7}=\left(\begin{array}{cc}
a & -1 \\
b-1 & 1
\end{array}\right), \quad A_{8}=\left(\begin{array}{cc}
-a & -1 \\
-b+1 & 1
\end{array}\right), \quad A_{9}=\left(\begin{array}{cc}
a-1 & -a \\
b & -b+1
\end{array}\right), \\
& A_{10}=\left(\begin{array}{cc}
a & -a+1 \\
b-1 & -b
\end{array}\right), \quad A_{11}=\left(\begin{array}{cc}
a-1 & a \\
b & b-1
\end{array}\right), \quad A_{12}=\left(\begin{array}{cc}
a & a-1 \\
b-1 & b
\end{array}\right) \\
& A_{12+k}:=-A_{k}, \quad k=1, \ldots, 12 .
\end{aligned}
$$

We are now able to state the main results of this paper:

Proposition 4.1. Each stair-like tile corresponding to a surrounding matrix $A=A_{i}(1 \leq i \leq 24)$ is a $\mathbb{Z}^{2}$-tile, i.e., each of these tiles induces a tiling of $\mathbb{R}^{2}$ by $\mathbb{Z}^{2}$-translates.

Proof. In the proof of Theorem 4.2 the graphs $G(R)$ are constructed explicitly for all these stair-like tiles. So, the criterion in Lemma 2.2 can be checked by direct calculation.

TheOREM 4.2. Let $\mathcal{T}=\mathcal{T}(A, \mathcal{D})$ be a stair-like tile and set $\mathcal{D}-\mathcal{D}:=\{d-$ $\left.d^{\prime} \mid d, d^{\prime} \in \mathcal{D}\right\}$. If $a, b \geq 2$ then $\mathcal{T}$ is disk-like if and only if one of the following conditions holds:
$A \in\left\{A_{1}, A_{2}, A_{3}, A_{13}, A_{14}, A_{15}\right\}$
$\wedge \quad\left(\begin{array}{l}0 \\ 2\end{array}\right) \notin \mathcal{D}-\mathcal{D}$,
$A \in\left\{A_{5}, A_{6}, A_{7}, A_{17}, A_{18}, A_{19}\right\}$
$\wedge \quad\left(\begin{array}{l}2 \\ 0\end{array}\right) \notin \mathcal{D}-\mathcal{D}$,
$A \in\left\{A_{9}, A_{21}\right\}$
$\wedge\left\{\begin{array}{lll}\left(\begin{array}{l}4 \\ 0\end{array}\right),\left(\begin{array}{l}0 \\ 4\end{array}\right) & \notin & \mathcal{D}-\mathcal{D} \\ \left\{\left(\begin{array}{l}a-1 \\ b-3\end{array}\right),\left(\begin{array}{l}a-3 \\ b-1\end{array}\right)\right\} & \not \subset & \mathcal{D}-\mathcal{D} \\ \left\{\left(\begin{array}{l}a-1 \\ b-4\end{array}\right),\left(\begin{array}{l}3 \\ 0\end{array}\right)\right\} & \not \subset & \mathcal{D}-\mathcal{D} \\ \left\{\left(\begin{array}{l}a-4 \\ b-1\end{array}\right),\left(\begin{array}{l}0 \\ 3\end{array}\right)\right\} & \not \subset & \mathcal{D}-\mathcal{D}\end{array}\right.$,
$A \in\left\{A_{10}, A_{22}\right\}$

$$
\wedge \quad\left\{\begin{aligned}
\left(\begin{array}{l}
4 \\
0
\end{array}\right),\left(\begin{array}{c}
0 \\
4
\end{array}\right) \notin \mathcal{D}-\mathcal{D} \\
\left(\begin{array}{l}
a-1 \\
b-3
\end{array}\right) \in \mathcal{D}-\mathcal{D} \Rightarrow \quad\left(\begin{array}{l}
0 \\
2
\end{array}\right) \notin \mathcal{D}-\mathcal{D} . \\
\left(\begin{array}{l}
a-3 \\
b-1
\end{array}\right) \in \mathcal{D}-\mathcal{D} \Rightarrow\left(\begin{array}{l}
2 \\
0
\end{array}\right) \notin \mathcal{D}-\mathcal{D} .
\end{aligned}\right.
$$

Remark 4.3. If $A \in\left\{A_{4}, A_{8}, A_{16}, A_{20}\right\}$ then the associated stair-like tile is never disk-like. If $A \in\left\{A_{11}, A_{12}, A_{23}, A_{24}\right\}$ then one of the eigenvalues of $A$ has modulus 1 . Thus for these matrices no $\mathbb{Z}^{2}$-tile can be defined. 
Remark 4.4. We formulated the theorem for $a, b \geq 2$ in order to avoid cumbersome notation in the formulation of our main result. Also the proof is given only for stair-like tiles satisfying $a, b \geq 2$ for the same reason. However, with slight adaptions our methods also yield a characterization result for the cases $a=1$ or $b=1$. The characterization of disk-like tiles for these special cases is the same as the characterization contained in Theorem 4.2 apart from the following exceptions:

- If $A \in\left\{A_{2}, A_{14}\right\}$ and $b=1$ then $\mathcal{T}(A, \mathcal{D})$ is disk-like if and only if $a \geq 4$.

- If $A \in\left\{A_{3}, A_{15}\right\}$ and $a=1$ then $\mathcal{T}(A, \mathcal{D})$ is never disk-like.

- If $A \in\left\{A_{4}, A_{16}\right\}$ and $b=1$ then $\mathcal{T}(A, \mathcal{D})$ is always disk-like.

- If $A \in\left\{A_{10}, A_{22}\right\}$ and $a=1$ or $b=1$ then one of the eigenvalues of $A$ has modulus 1 .

5. Proof of the theorem. Due to Lemma 2.5 we know that $S(A, \mathcal{D})=$ $S(-A, \mathcal{D})$ holds. So we may confine ourselves to investigate the tiles $\mathcal{T}\left(A_{i}, \mathcal{D}(P)\right)$ for $i=1, \ldots, 12$. In addition, consider the reflection $\star: \mathbb{R}^{2} \rightarrow \mathbb{R}^{2}:\left(\begin{array}{l}x_{1} \\ x_{2}\end{array}\right) \mapsto\left(\begin{array}{l}x_{2} \\ x_{1}\end{array}\right)$. With $B:=\left(\begin{array}{ll}b_{11} & b_{12} \\ b_{21} & b_{22}\end{array}\right)$ and $B^{\star}:=\left(\begin{array}{ll}b_{22} & b_{21} \\ b_{12} & b_{11}\end{array}\right)$ we get $(B \boldsymbol{x})^{\star}=B^{\star} \boldsymbol{x}^{\star}$ which leads to $\mathcal{T}(B, \mathcal{D}(P))^{\star}=\mathcal{T}\left(B^{\star}, \mathcal{D}(P)^{\star}\right)$. Now, $P$ is stair-like and so is the polyomino $P^{\star}$ determined by $\mathcal{D}(P)^{\star}=\left\{\left(\begin{array}{l}0 \\ 0\end{array}\right), \ldots,\left(\begin{array}{c}b-1 \\ a-1\end{array}\right)\right\}$.

As the parameters $a, b \geq 2$ may be chosen arbitrarily, we may carry over the results from the matrices $A_{1}, \ldots, A_{4}$ to $A_{5}, \ldots, A_{8}$ via $\star$ and work is cut in half.

The matrices $A_{11}$ and $A_{12}$ have eigenvalues of modulus 1 and therefore do not deserve interest. This means that we are going to check the disk-likeness of the tiles $\mathcal{T}\left(A_{i}, \mathcal{D}(P)\right)$ with $i \in\{1,2,3,4,9,10\}$. From these results we can deduce the outcomes for all other cases $\mathcal{T}\left(A_{i}, \mathcal{D}(P)\right)$ with $i \notin\{1,2,3,4,9,10\}$.

Given a tile $\mathcal{T}=\mathcal{T}\left(A_{i}, \mathcal{D}(P)\right)$ we primarily compute the set $S$ of its neighbors. The starting point for the construction of $S$ is the graph $G(R)$ where $R=R(A, \mathcal{D})$ is defined as in (2.2).

Lemma 5.1. For every pair $\left(A_{i}, \mathcal{D}\right)$ where $\mathcal{D}$ defines a stair-like polyomino and $A_{i}$ is one of its surrounding matrices we get $\left|G_{A_{i}}(R)\right|=7$.

This can be seen as follows:

We know that $A$ is a surrounding matrix of the polyomino $P$ defined by $\mathcal{D}$. This is why we get $\left\{A x \mid x \in\left\{ \pm e_{1}, \pm e_{2}, \pm e\right\}\right\}=\{ \pm(1,-1), \pm(a, b-1), \pm(a-1, b)\}$. The vector $e$ depends on the special choice of the matrix $A$ and is defined as $e:=e_{1}+e_{2}$ in case of $A\left(e_{1}+e_{2}\right) \in\{ \pm(1,-1), \pm(a, b-1), \pm(a-1, b)\}$, or $e:=e_{1}-e_{2}$ otherwise. The central polyomino $P$ has a hexagonal surrounding and its six neighbors are the polyominoes $A x+P$ where $x \in\left\{ \pm e_{1}, \pm e_{2}, \pm e\right\}$.

We start with the construction of the set $R$ according to formula (2.1). With $R_{0}=\left\{0, \pm e_{1}, \pm e_{2}\right\}$ the set $R_{1}:=R_{0} \cup\left\{k \in \mathbb{Z}^{2} \mid(A k+\mathcal{D}) \cap(l+\mathcal{D}) \neq \emptyset\right.$ for $\left.l \in R_{0}\right\}$ has to be computed. We have a closer look at the intersection $(A k+\mathcal{D}) \cap(l+\mathcal{D})$. The set $l+\mathcal{D}$ determines the polyomino $l+P$ which is a translated copy of $P$. More precisely, $P$ is translated at most by one Voronoi cell in horizontal or vertical direction, because $l \in R_{0}$. So $(A k+\mathcal{D}) \cap(l+\mathcal{D}) \neq \emptyset$ if and only if $A k+P$ equals $P$ or is a neighbor of $P$. We already know that this means $A k \in\{(0,0), \pm(1,-1), \pm(a, b-1), \pm(a-1, b)\}$ or $k \in\left\{0, \pm e_{1}, \pm e_{2}, \pm e\right\}$ respectively. Summing up, this gives $R_{1}=\left\{0, \pm e_{1}, \pm e_{2}, \pm e\right\}$.

In the next iteration step we have to characterize the set $R_{2}=R_{1} \cup\{k \in$ $\mathbb{Z}^{2} \mid(A k+\mathcal{D}) \cap(l+\mathcal{D}) \neq \emptyset$ for $\left.l \in R_{1}\right\}$. As before the translated copies of $P$ are decisive. 
As $l \in R_{1}$, the polyomino $P$ is translated at most by one Voronoi cell in diagonal, horizontal or vertical direction. So $l+P$ where $l \in R_{1}$ just meets the neighbors of $P$. This means that $(A k+\mathcal{D}) \cap(l+\mathcal{D}) \neq \emptyset$ if and only if $k \in\left\{0, \pm e_{1}, \pm e_{2}, \pm e\right\}$.

We directly read off that $R_{2}=R_{1}$ holds. So the iteration process for the construction of $R$ terminates and we get $R=R_{1}=\left\{0, \pm e_{1}, \pm e_{2}, \pm e\right\}$. Figure 4 illustrates the situation in case of $A=A_{1}=\left(\begin{array}{cc}1 & a \\ -1 & b-1\end{array}\right)$ with $a=6$ and $b=3$. For this example we have $A\left( \pm e_{1}\right)= \pm(1,-1), A\left( \pm e_{2}\right)= \pm(a, b-1)$ and $A( \pm e)=\mp(a-1, b)$ with $e:=e_{1}-e_{2}$.

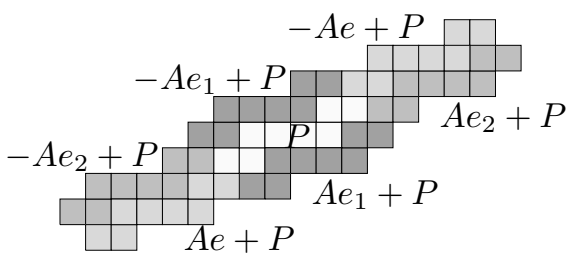

FIG. 4. Neighborhood of a stair-like polyomino

Remark 5.2. We are now in the situation to construct the graph $G_{A_{i}, \mathcal{D}}(R)$ for given $A_{i}$ and $\mathcal{D}$ very easily. The set of vertices of $G_{A_{i}, \mathcal{D}}(R)$ is $R=\left\{0, \pm e_{1}, \pm e_{2}, \pm e\right\}$ where $e \in\left\{e_{1}+e_{2}, e_{1}-e_{2}\right\}$ is defined such that $A_{i} e \in\{ \pm(1,-1), \pm(a, b-1), \pm(a-1, b)\}$. Moreover, there exists an edge from $v_{1} \in R$ to $v_{2} \in R$ labelled by $d \mid d^{\prime}$ if and only if $A_{i} v_{1}+d^{\prime}=v_{2}+d$.

The proof of the theorem now runs as follows. Given a matrix $A_{i}$ we first construct the graph $G_{A_{i}, \mathcal{D}}(R)$ according to the above remark. In a second step we apply Algorithm 2.4 to generate $G_{A_{i}, \mathcal{D}}(S)$ and compute the set $S$ of neighbors of the tile $\mathcal{T}=\mathcal{T}\left(A_{i}, \mathcal{D}\right)$. With the help of Proposition 2.6, Lemma 2.8 and Lemma 2.9 it is then straightforward to decide whether $\mathcal{T}$ is disk-like or not.

Remark 5.3. In view of Proposition 2.6 we may interrupt Algorithm 2.4, as soon as we know that $\left|G_{A_{i}, \mathcal{D}}(S)\right| \geq 10$ holds. Then the tile $\mathcal{T}$ is not disk-like.

Remark 5.4. In the case of a hexagonal surrounding, i.e., $R \backslash\{0\}=S$, we always get a disk-like tile $\mathcal{T}$. This follows from the shape of a polyomino $P$, as the associated set $\mathcal{D}=\mathcal{D}(P)$ is always $\left\{ \pm e_{1}, \pm e_{2}, \pm e\right\}$-connected.

In what follows, we are not interested in the exact shape or number of edges leading from one state to another. Thus, if there exist (one or more) edges $v \stackrel{d_{1} \mid d_{2}}{\longrightarrow} v^{\prime}$ with $d_{1}-d_{2}=d$ in a graph, we collect these edges to a single edge and label it with $v \stackrel{d}{\rightarrow} v^{\prime}$. With this notation the edges $v_{1} \stackrel{\ell_{1}}{\longrightarrow} v_{1}^{\prime}$ in $G_{1}$ and $v_{2} \stackrel{\ell_{2}}{\longrightarrow} v_{2}^{\prime}$ in $G_{2}$ lead to an edge $v_{1}+v_{2} \stackrel{\ell_{1}+\ell_{2}}{\longrightarrow} v_{1}^{\prime}+v_{2}^{\prime}$ in $G_{1} \otimes G_{2}$ provided that $\ell_{1}+\ell_{2} \in \mathcal{D}-\mathcal{D}$.

5.1. First type of matrix. Figure 5 shows the graph $G(R)$ stemming from a stair-like tile $\mathcal{T}\left(A_{1}, \mathcal{D}\right)$ with surrounding matrix $A_{1}=\left(\begin{array}{cc}1 & a \\ -1 & b-1\end{array}\right)$. We apply Algorithm 2.4 to determine $G(S)$. In the fifth line of the algorithm we construct the product-graph $B[1] \otimes B[1]$ that equals $G(R) \otimes G(R)$ as $B[1]=\operatorname{Red}(G(R))=G(R)$. 


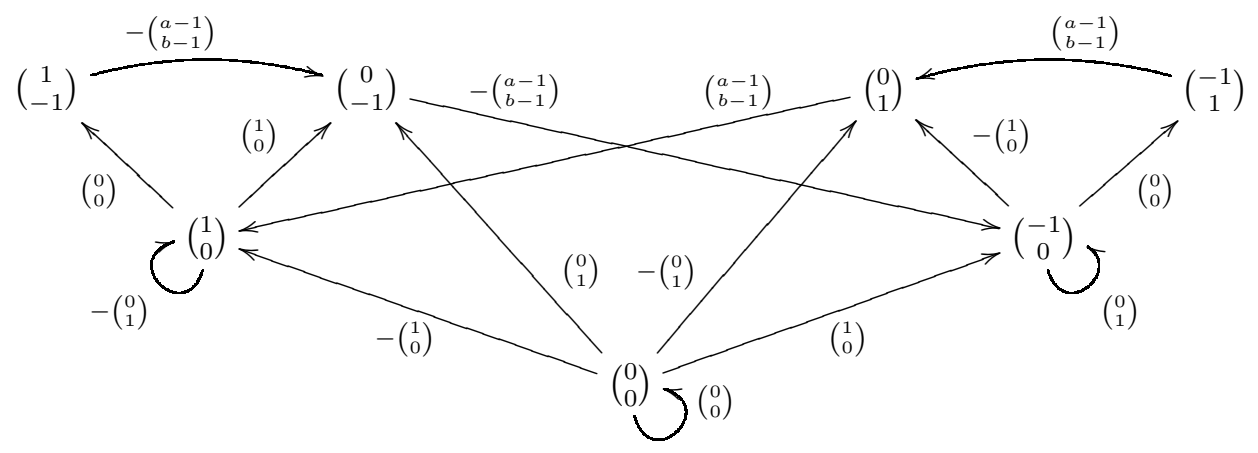

FIG. 5. First graph

According to Definition 2.3 the states of $G(R) \otimes G(R)$ are of the form $r_{2}=r_{1}+r_{1}^{\prime}$ with $r_{1}, r_{1}^{\prime} \in G(R)$.

We are mainly interested in the states of $G(R) \otimes G(R)$ that are not contained in $G(R)$, in this case these are the states $\pm(2,-2), \pm(1,-2), \pm(0,-2), \pm(2,-1), \pm(2,0)$, $\pm(1,1)$. In view of the remark after Lemma 2.5 we only have to examine the vertices with "+"-sign in front of the brackets.

Suppose that $\left(\begin{array}{l}0 \\ 2\end{array}\right) \in \mathcal{D}-\mathcal{D}$ holds. Then there are elements $d_{1}, d_{2} \in \mathcal{D}$ with $d_{2}-d_{1}=$ $\left(\begin{array}{l}0 \\ 2\end{array}\right)$. As we exclusively deal with polyominoes, we also have $d_{1}^{\prime}:=d_{1}+\left(\begin{array}{l}0 \\ 1\end{array}\right) \in \mathcal{D}$. By the definition of $G(R)$ the edge $(1,0) \stackrel{-\left(\begin{array}{l}0 \\ 1\end{array}\right)}{\longrightarrow}(1,0)$ in Figure 5 is a shortcut containing the edges labelled by $d_{1} \mid d_{1}^{\prime}$ and $d_{1}^{\prime} \mid d_{2}$. Following Definition 2.3 the product-graph $G(R) \otimes G(R)$ contains the edge $(1,0)+(1,0) \stackrel{d_{1} \mid d_{2}}{\longrightarrow}(1,0)+(1,0)$, which obviously is a cycle, namely $(2,0) \stackrel{-\left(\begin{array}{c}0 \\ 2\end{array}\right)}{\longrightarrow}(2,0)$. Of course, from now on the vertex $(2,0)$ is not removed during the algorithm, as it is a starting point of a walk of infinite length.

Until now we know $\{ \pm(1,0), \pm(0,1), \pm(1,-1), \pm(2,0)\} \subset G(S)$. In view of Proposition 2.6 and Lemma 2.9 there should exist $\alpha, \beta \in \mathbb{Z}^{2}$ such that

$$
\{ \pm(1,0), \pm(0,1), \pm(1,-1), \pm(2,0)\}=\{ \pm \alpha, \pm \beta, \pm(\alpha+\beta), \pm(\alpha-\beta)\}
$$

in case of a disk-like tile $\mathcal{T}\left(A_{1}, \mathcal{D}\right)$. A short calculation shows that this is not possible.

Keep in mind that this argumentation $\left(\left(\begin{array}{l}0 \\ 2\end{array}\right) \in \mathcal{D}-\mathcal{D} \Rightarrow \mathcal{T}\right.$ not disk-like $)$ is applicable for all tiles stemming from the matrices $A_{1}, \ldots, A_{4}$. Thus, from now on we may assume that $\left(\begin{array}{l}0 \\ 2\end{array}\right) \notin \mathcal{D}-\mathcal{D}$ holds.

According to Algorithm 2.4 we compute $\operatorname{Red}(B[1] \otimes B[1])$, i.e., we successively delete those states that are not starting points of a walk of infinite length.

The only edge leaving $(1,-1)$ in $G(R)$ is $(1,-1) \stackrel{-\left(\begin{array}{c}a-1 \\ b-1\end{array}\right)}{\longrightarrow}(0,-1)$. As $(2,-2)=$ $(1,-1)+(1,-1)$ is the only way of writing $(2,-2)$ as sum of two elements of $R$ the only possible edge leaving $(2,-2)$ in $B[1] \otimes B[1]$ is $(2,-2) \stackrel{-\left(\begin{array}{c}2 a-2 \\ 2 b-2\end{array}\right)}{\longrightarrow}(0,-2)$. But this is not feasible as $-\left(\begin{array}{c}2 a-2 \\ 2 b-2\end{array}\right) \notin \mathcal{D}-\mathcal{D}$ (note that $a, b \geq 2$ ) and so we remove $\pm(2,-2)$. A similar reasoning allows to delete $\pm(1,-2), \pm(0,-2)$.

Now, the vertex $(2,-1)=(1,-1)+(1,0)$ is our object of scrutiny. The only way of writing $(2,-1)$ as sum of two elements of $R$ is $(1,-1)+(1,0)$. Thus we focus on all 
edges leaving the vertices $(1,-1)$ and $(1,0)$ in $G(R)$, namely $(1,-1) \stackrel{-\left(\begin{array}{c}a-1 \\ b-1\end{array}\right)}{\longrightarrow}(0,-1)$, $(1,0) \stackrel{\left(\begin{array}{l}0 \\ 0\end{array}\right)}{\longrightarrow}(1,-1),(1,0) \stackrel{\left(\begin{array}{l}1 \\ 0\end{array}\right)}{\longrightarrow}(0,-1)$ and $(1,0) \stackrel{-\left(\begin{array}{l}0 \\ 1\end{array}\right)}{\longrightarrow}(1,0)$. Thus, edges leaving $(2,-1)$ might lead to the vertices $(1,-2),(0,-2)$ and $(1,-1)$. However, $(1,-2)$ and $(0,-2)$ are already deleted and there is no edge $(2,-1) \stackrel{-\left(\begin{array}{c}a-1 \\ b\end{array}\right)}{\longrightarrow}(1,-1)$ as we have $-\left(\begin{array}{c}a-1 \\ b\end{array}\right) \notin \mathcal{D}-\mathcal{D}$. Hence, we delete $\pm(2,-1)$.

Now we have a closer look at the edges leaving $(2,0)$. Possible destinations for these edges are the states $s_{2}=s_{1}+s_{1}^{\prime}$ with $s_{1}, s_{1}^{\prime} \in\{(1,0),(0,-1),(1,-1)\}$. Apart from $(2,0)$ and $(1,-1)$, all of them have already been removed.

As we assumed $\left(\begin{array}{l}0 \\ 2\end{array}\right) \notin \mathcal{D}-\mathcal{D}$, there is no edge $(2,0) \stackrel{-\left(\begin{array}{c}0 \\ 2\end{array}\right)}{\longrightarrow}(2,0)$. Likewise the edge $(2,0) \stackrel{\left(\begin{array}{c}1 \\ -1\end{array}\right)}{\longrightarrow}(1,-1)$ is not possible due to $\left(\begin{array}{c}1 \\ -1\end{array}\right) \notin \mathcal{D}-\mathcal{D}$. So we delete $\pm(2,0)$.

Finally, edges starting at $(1,1)$ in $B[1] \otimes B[1]$ may lead to $(2,0),(2,-1)$ or $(1,-1)$, respectively. However, the first and second vertex are already deleted and the third is not possible as $\left(\begin{array}{c}a \\ b-1\end{array}\right) \notin \mathcal{D}-\mathcal{D}$.

At this moment, Algorithm 2.4 leaves the loop as $B[2]=B[1]$ holds. We have $|G(S)|=6$ and Remark 5.4 tells us that the arising $\mathbb{Z}^{2}$-tile $\mathcal{T}$ is disk-like.

5.2. Second type of matrix. We now investigate the graph $G(R)$ associated to a $\mathbb{Z}^{2}$-tile $\mathcal{T}\left(A_{2}, \mathcal{D}\right)$ with $A_{2}=\left(\begin{array}{cc}1 & -a \\ -1 & -(b-1)\end{array}\right) . G(R)$ is depicted in Figure 6 (dotted lines only occur for tiles for that their label is contained in $\mathcal{D}-\mathcal{D}$ ). Again, we construct the graph $G(S)$ by the help of Algorithm 2.4.

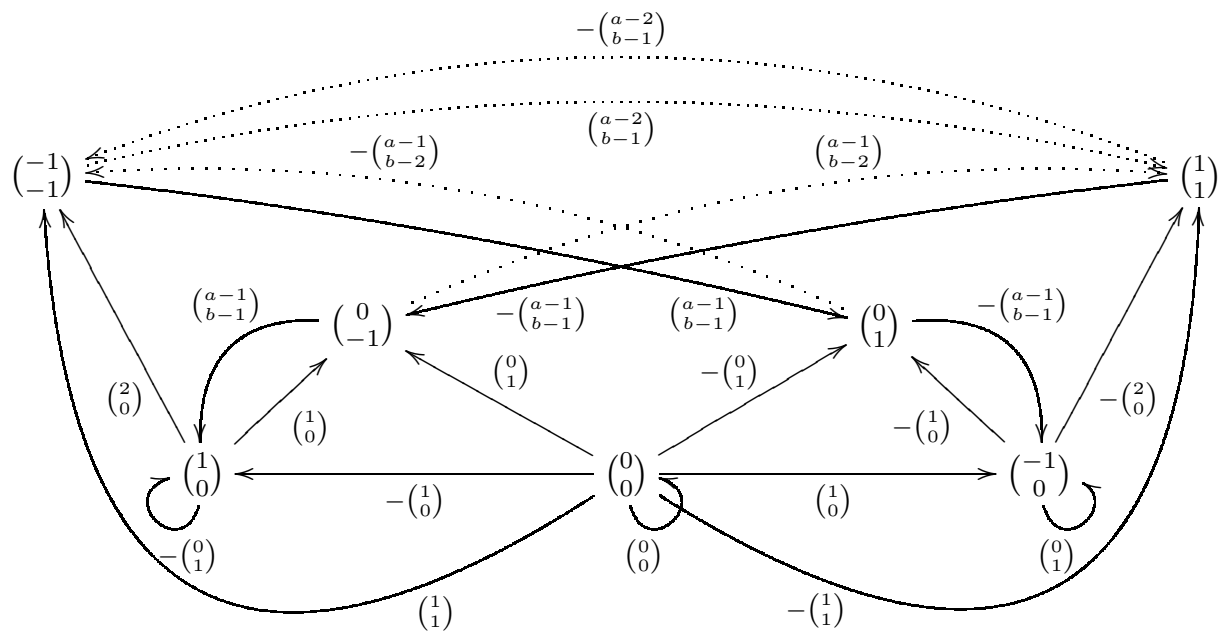

FIG. 6. Second graph

The following states of the product-graph $B[1] \otimes B[1]=G(R) \otimes G(R)$ are not contained in $G(R): \pm(2,2), \pm(1,2), \pm(0,2), \pm(2,1), \pm(2,0), \pm(1,-1)$. As in the previous subsection, we suppose $\left(\begin{array}{l}0 \\ 2\end{array}\right) \notin \mathcal{D}-\mathcal{D}$ because otherwise $\pm(2,0) \in G(S)$ implies that $\mathcal{T}$ is not disk-like.

Possible edges leaving from $(2,2)=(1,1)+(1,1)$ are $(2,2) \stackrel{-\left(\begin{array}{c}2 a-4 \\ 2 b-2\end{array}\right)}{\longrightarrow}(-2,-2)$, 
$(2,2) \stackrel{-\left(\begin{array}{c}2 a-3 \\ 2 b-2\end{array}\right)}{\longrightarrow}(-1,-2)$ and $(2,2) \stackrel{-\left(\begin{array}{c}2 a-2 \\ 2 b-2\end{array}\right)}{\longrightarrow}(0,-2)$. However, all the labels are not elements of $\mathcal{D}-\mathcal{D}$ and so the vertices $\pm(2,2)$ are removed. A similar reasoning shows that $\pm(1,2)$ and $\pm(0,2)$ have to be deleted.

Furthermore, edges leaving $(2,1)=(1,1)+(1,0)$ may lead to $(-2,-2),(-1,-2)$, $(0,-2),(1,-1)$ and $(0,-1)$, respectively. However, the reduction process has already deleted $\pm(2,2), \pm(1,2)$ and $\pm(0,2)$. So only the edges $(2,1) \stackrel{-\left(\begin{array}{c}a-1 \\ b\end{array}\right)}{\longrightarrow}(1,-1)$ and $(2,1) \stackrel{-\left(\begin{array}{c}a-2 \\ b\end{array}\right)}{\longrightarrow}(0,-1)$ have to be checked. The labels $-\left(\begin{array}{c}a-1 \\ b\end{array}\right)$ and $-\left(\begin{array}{c}a-2 \\ b\end{array}\right)$ are not elements of $\mathcal{D}-\mathcal{D}$. Thus there are no edges leaving the vertices $\pm(2,1)$ and so we remove them.

The remaining states are $\pm(2,0)$ and $\pm(1,-1)$. Possible targets for edges leaving $(2,0)$ are the vertices $(-2,-2),(-1,-2),(0,-2),(1,-1),(0,-1)$ and $(2,0)$. However, $(-2,-2),(-1,-2)$ and $(0,-2)$ are already removed. Moreover, the edges $(2,0) \stackrel{\left(\begin{array}{c}1 \\ -1\end{array}\right)}{\longrightarrow}$ $(1,-1),(2,0) \stackrel{\left(\begin{array}{c}2 \\ -1\end{array}\right)}{\longrightarrow}(0,-1)$ and $(2,0) \stackrel{-\left(\begin{array}{c}0 \\ 2\end{array}\right)}{\longrightarrow}(2,0)$ do not exist as the labels are not elements of $\mathcal{D}-\mathcal{D}$. As a consequence we remove $\pm(2,0)$.

Finally we focus on edges leaving $(1,-1)$ which may lead to $(2,1),(2,0),(1,-1)$, $(0,0),(0,-1)$ and $(1,0)$. We realize that the vertices $(2,1),(2,0)$ are already removed. The edges $(1,-1) \stackrel{\left(\begin{array}{c}a \\ b-1\end{array}\right)}{\longrightarrow}(1,-1),(1,-1) \stackrel{\left(\begin{array}{l}a+1 \\ b-2\end{array}\right)}{\longrightarrow}(0,0),(1,-1) \stackrel{\left(\begin{array}{l}a+1 \\ b-1\end{array}\right)}{\longrightarrow}(0,-1)$ and $(1,-1) \stackrel{\left(\begin{array}{c}a \\ b-2\end{array}\right)}{\longrightarrow}(1,0)$ do not exist as the labels do not belong to $\mathcal{D}-\mathcal{D}$. Thus, we remove $\pm(1,-1)$ and get $|G(S)|=6$.

At this moment, we know that the resulting $\mathbb{Z}^{2}$-tile $\mathcal{T}$ is disk-like (cf. Remark 5.4).

5.3. Third type of matrix. In this subsection the starting point are $\mathbb{Z}^{2}$-tiles $\mathcal{T}\left(A_{3}, \mathcal{D}\right)$ with $A_{3}=\left(\begin{array}{cc}1 & a-1 \\ -1 & b\end{array}\right)$. The associated graph $G(R)$ is depicted in Figure 7. It turns out that this case is a bit more involved than the previous ones.

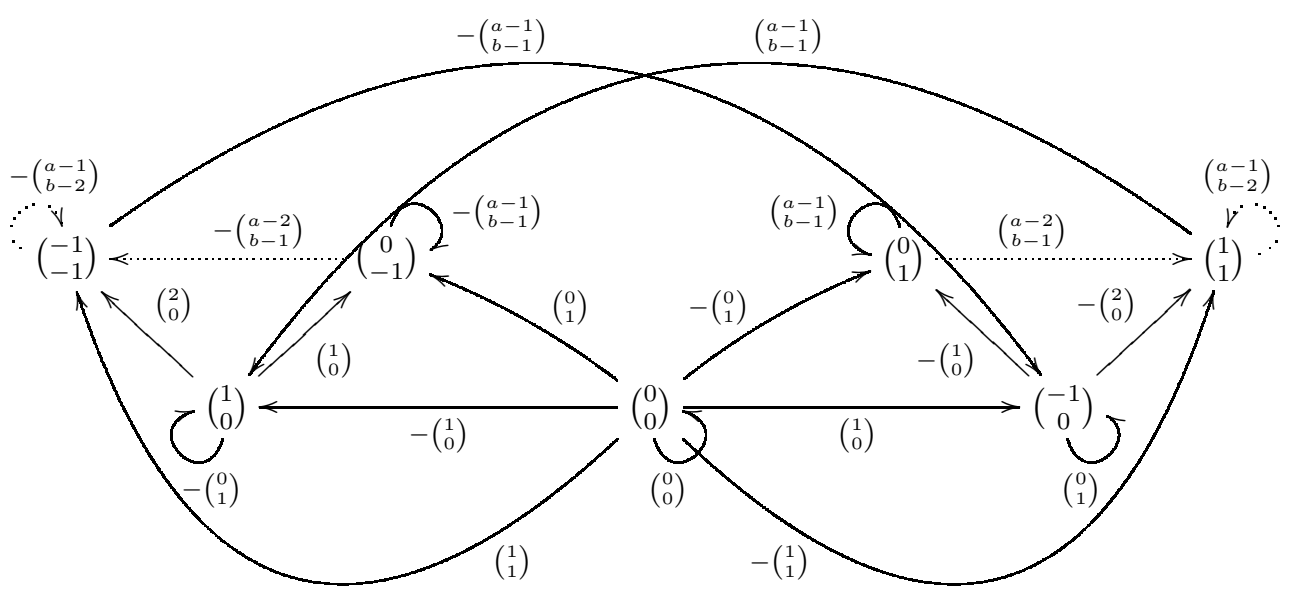

FIG. 7. Third graph

The following states of the product-graph $B[1] \otimes B[1]=G(R) \otimes G(R)$ are not 
contained in $G(R): \pm(2,2), \pm(0,2), \pm(1,2), \pm(2,0), \pm(1,-1), \pm(2,1)$. Similar to the previous subsection, the vertices $\pm(2,2), \pm(0,2)$ are removed as edges starting there would require that some of the labels $\left(\begin{array}{c}2 a-2 \\ 2 b-2\end{array}\right),\left(\begin{array}{c}2 a-2 \\ 2 b-3\end{array}\right),\left(\begin{array}{c}2 a-2 \\ 2 b-4\end{array}\right),\left(\begin{array}{c}2 a-3 \\ 2 b-2\end{array}\right)$ and $\left(\begin{array}{l}2 a-4 \\ 2 b-2\end{array}\right)$ were elements of $\mathcal{D}-\mathcal{D}$, which is not the case.

Due to the same reason there is at most one edge starting at $(1,2)$, namely $(1,2) \stackrel{\left(\begin{array}{l}2 a-3 \\ 2 b-3\end{array}\right)}{\longrightarrow}(2,2)$. However, the state $(2,2)$ is already removed and therefore we delete $\pm(1,2)$.

Analogous to the previous subsections, we recall the assumption $\left(\begin{array}{l}0 \\ 2\end{array}\right) \notin \mathcal{D}-\mathcal{D}$ which, also in this case, is a necessary condition for the tile to be disk-like.

For paths starting at $(2,0)$ there are six possible targets: $(-2,-2),(-1,-2)$, $(0,-2),(2,0),(1,-1),(0,-1)$. However, the first 3 states have just been extinguished, and the last 3 vertices are not feasible as all of the edges $(2,0) \stackrel{\left(\begin{array}{c}1 \\ -1\end{array}\right)}{\longrightarrow}(1,-1)$, $(2,0) \stackrel{\left(\begin{array}{c}2 \\ -1\end{array}\right)}{\longrightarrow}(0,-1)$ and $(2,0) \stackrel{-\left(\begin{array}{c}0 \\ 2\end{array}\right)}{\longrightarrow}(2,0)$ have labels that do not belong to $\mathcal{D}-\mathcal{D}$. Thus, we delete $\pm(2,0)$.

Edges starting at $(1,-1)$ can lead to $(-2,-2),(-1,-2),(0,-2),(1,-1)$ and $(0,-1)$. The first 3 vertices are already removed and so the edges $(1,-1) \stackrel{-\left(\begin{array}{c}a-1 \\ b\end{array}\right)}{\longrightarrow}$ $(1,-1)$ and $(1,-1) \stackrel{-\left(\begin{array}{c}a-2 \\ b\end{array}\right)}{\longrightarrow}(0,-1)$ are to be analyzed. However, the labels $-\left(\begin{array}{c}a-1 \\ b\end{array}\right)$ and $-\left(\begin{array}{c}a-2 \\ b\end{array}\right)$ are not elements of $\mathcal{D}-\mathcal{D}$ and so the vertices $\pm(1,-1)$ are removed.

Finally, the investigation of the states $\pm(2,1)$ is left over. We have a look at all possible edges leaving $(2,1)$. Their endpoints may be the following: $(2,0),(1,-1)$, $(0,-1),(1,0),(0,0)$ and $(2,1)$. Keep in mind that the states $(2,0)$ and $(1,-1)$ are already removed. We check the edges $(2,1) \stackrel{\left(\begin{array}{c}a+1 \\ b-1\end{array}\right)}{\longrightarrow}(0,-1),(2,1) \stackrel{\left(\begin{array}{c}a \\ b-2\end{array}\right)}{\longrightarrow}(1,0)$, $(2,1) \stackrel{\left(\begin{array}{c}a+1 \\ b-2\end{array}\right)}{\longrightarrow}(0,0)$ and $(2,1) \stackrel{\left(\begin{array}{c}a-1 \\ b-3\end{array}\right)}{\longrightarrow}(2,1)$. As the labels $\left(\begin{array}{l}a+1 \\ b-1\end{array}\right),\left(\begin{array}{c}a \\ b-2\end{array}\right),\left(\begin{array}{l}a+1 \\ b-2\end{array}\right)$ do not belong to $\mathcal{D}-\mathcal{D}$, edges with endpoints $(0,-1),(1,0)$ and $(0,0)$ do not exist. The edge $(2,1) \stackrel{\left(\begin{array}{c}a-1 \\ b-3\end{array}\right)}{\longrightarrow}(2,1)$ exists, if and only if $\left(\begin{array}{l}a-1 \\ b-3\end{array}\right) \in \mathcal{D}-\mathcal{D}$. Thus we have two possibilities: (A) The label $\left(\begin{array}{l}a-1 \\ b-3\end{array}\right)$ is not contained in $\mathcal{D}-\mathcal{D}$. Then the states $\pm(2,1)$ are removed and Algorithm 2.4 terminates. We get $|G(S)|=6$ and know that the arising reptile is disk-like (cf. Remark 5.4).

(B) The point $\left(\begin{array}{l}a-1 \\ b-3\end{array}\right)$ is an element of $\mathcal{D}-\mathcal{D}$. Then we have the cycles $\pm(2,1) \stackrel{ \pm\left(\begin{array}{l}a-1 \\ b-3\end{array}\right)}{\longrightarrow}$ $\pm(2,1)$ and $B[2] \neq B[1]$ in Algorithm 2.4. We compute the graph-product $B[2] \otimes$ $B[1]$ and have a closer look at the states $\pm(3,2), \pm(2,0), \pm(2,2), \pm(3,1)$ that are not elements of $B[2]=\operatorname{Red}(B[1] \otimes B[1])$. As $\left(\begin{array}{c}2 a-2 \\ 2 b-4\end{array}\right)$ and $\left(\begin{array}{c}2 a-2 \\ 2 b-5\end{array}\right)$ do not belong to $\mathcal{D}-\mathcal{D}$ there is no edge leaving $\pm(3,2)$ and so we remove $\pm(3,2)$. Besides, the vertices $\pm(2,0)$ may also be deleted as both of the edges $(2,0) \stackrel{\left(\begin{array}{c}1 \\ -2\end{array}\right)}{\longrightarrow}(1,0)$ and $(2,0) \stackrel{-\left(\begin{array}{l}0 \\ 2\end{array}\right)}{\longrightarrow}(2,0)$ are not possible due to $\left(\begin{array}{c}1 \\ -2\end{array}\right),\left(\begin{array}{l}0 \\ 2\end{array}\right) \notin \mathcal{D}-\mathcal{D}$. Now we focus on the state $(2,2)$. The edge $(2,2) \stackrel{\left(\begin{array}{c}2 a-2 \\ 2 b-4\end{array}\right)}{\longrightarrow}(2,2)$ is not feasible as $\left(\begin{array}{c}2 a-2 \\ 2 b-4\end{array}\right) \notin \mathcal{D}-\mathcal{D}$. Moreover, the state $(3,2)$ is already removed and so there cannot be an edge from $(2,2)$ to $(3,2)$. Summing up, there is no edge that leaves $(2,2)$ and therefore the states $\pm(2,2)$ are removed. Finally, we analyze the edges that leave $(3,1)$. We recognize that $(3,1) \stackrel{\left(\begin{array}{l}a-1 \\ b-4\end{array}\right)}{\longrightarrow}(3,1)$ is the only edge that seems to be possible at first sight. Other alternatives drop out because the required labels do not belong to $\mathcal{D}-\mathcal{D}$. However, we recall the assumption $\left(\begin{array}{l}0 \\ 2\end{array}\right) \notin \mathcal{D}-\mathcal{D}$ that results in $\left(\begin{array}{l}a-1 \\ b-4\end{array}\right) \notin \mathcal{D}-\mathcal{D}$. 
Thus Algorithm 2.4 terminates and we get $S=\{ \pm(1,0), \pm(0,1), \pm(1,1), \pm(2,1)\}$. With the notation of Lemma 2.9 we have $\alpha=(1,1), \beta=(1,0)$. The set $\mathcal{D}$ is $\{ \pm \alpha, \pm \beta\}$-connected if and only if $(0,2) \notin \mathcal{D}-\mathcal{D}$ which holds troughout this subsection.

So we get a parallelogramic surrounding with a disk-like tile.

5.4. Fourth type of matrix. We consider $\mathbb{Z}^{2}$-tiles $\mathcal{T}\left(A_{4}, \mathcal{D}\right)$ with $A_{4}=$ $\left(\begin{array}{cc}1 & -(a-1) \\ -1 & -b\end{array}\right)$ and get the graph $G(R)$ in Figure 8.

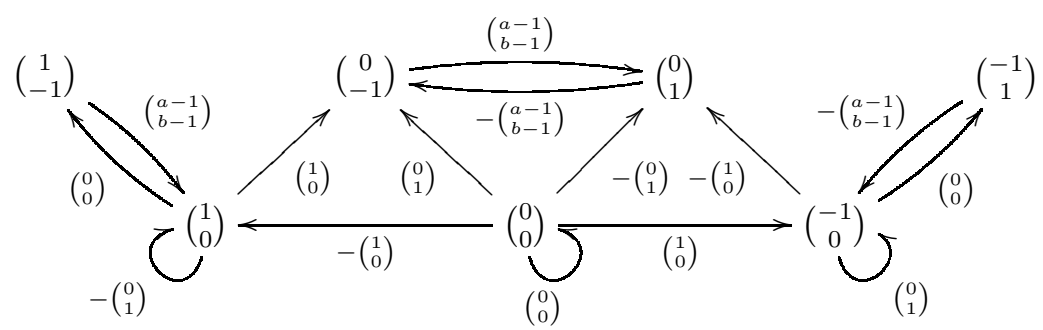

FIG. 8. Fourth graph

A short look at the product-graph $B[1] \otimes B[1]=G(R) \otimes G(R)$ shows that it contains the edges $(2,-1) \stackrel{\left(\begin{array}{c}a-1 \\ b-1\end{array}\right)}{\longrightarrow}(2,-1)$ and $(2,0) \stackrel{\left(\begin{array}{c}0 \\ -1\end{array}\right)}{\longrightarrow}(2,-1)$.

As the first edge is a cycle and the second ends in it, the states $(2,0),(2,-1)$ and their counterparts $-(2,0),-(2,-1)$ are elements of the graph $G(S)$. So we immediately deduce $|G(S)| \geq 10$ and according to Proposition 2.6 the $\mathbb{Z}^{2}$-tile $\mathcal{T}$ is not disk-like.

5.5. Fifth type of matrix. The $\mathbb{Z}^{2}$-tile $\mathcal{T}\left(A_{9}, \mathcal{D}\right)$ with matrix $A_{9}=$ $\left(\begin{array}{cc}a-1 & -a \\ b & -(b-1)\end{array}\right)$ yields the graph $G(R)$ in Figure 9. For this matrix the topological behaviour of the associated tile depends even more on the shape of the set $\mathcal{D}$ than in the previous cases.

We apply Algorithm 2.4 to determine the graph $G(S)$. The following states of the graph $B[1] \otimes B[1]=G(R) \otimes G(R)$ are not elements of $G(R): \pm(2,0), \pm(0,2)$, $\pm(1,-1), \pm(1,2), \pm(2,1), \pm(2,2)$. Similar to the previous subsections it is clear that $\pm(2,0), \pm(0,2)$ do not belong to $\operatorname{Red}(B[1] \otimes B[1])$, as no edge leaves these states. This can be seen very easily as the labels $\left(\begin{array}{l}2 a-2 \\ 2 b-2\end{array}\right),\left(\begin{array}{l}2 a-3 \\ 2 b-2\end{array}\right),\left(\begin{array}{c}2 a-4 \\ 2 b-2\end{array}\right),\left(\begin{array}{c}2 a-2 \\ 2 b-3\end{array}\right)$ and $\left(\begin{array}{l}2 a-2 \\ 2 b-4\end{array}\right)$ are not elements of $\mathcal{D}-\mathcal{D}$.

This is also the reason for there is at most one edge leaving the state $(1,-1)$, namely $(1,-1) \stackrel{\left(\begin{array}{l}2 a-3 \\ 2 b-3\end{array}\right)}{\longrightarrow}(2,2)$. Note that this exactly occurs when $a=b=2$. We will soon recognize that $\pm(2,2) \in G(S)$ implies that the associated tile is not disk-like. So we focus on the case where $\pm(2,2) \notin G(S)$ holds. However, in this case there is no edge leaving $\pm(1,-1)$ and so these states are removed.

For the remaining vertices $\pm(1,2), \pm(2,1), \pm(2,2)$ we have to analyze the situation. We list all edges that leave the states $(1,2),(2,1),(2,2)$ and lead to vertices 


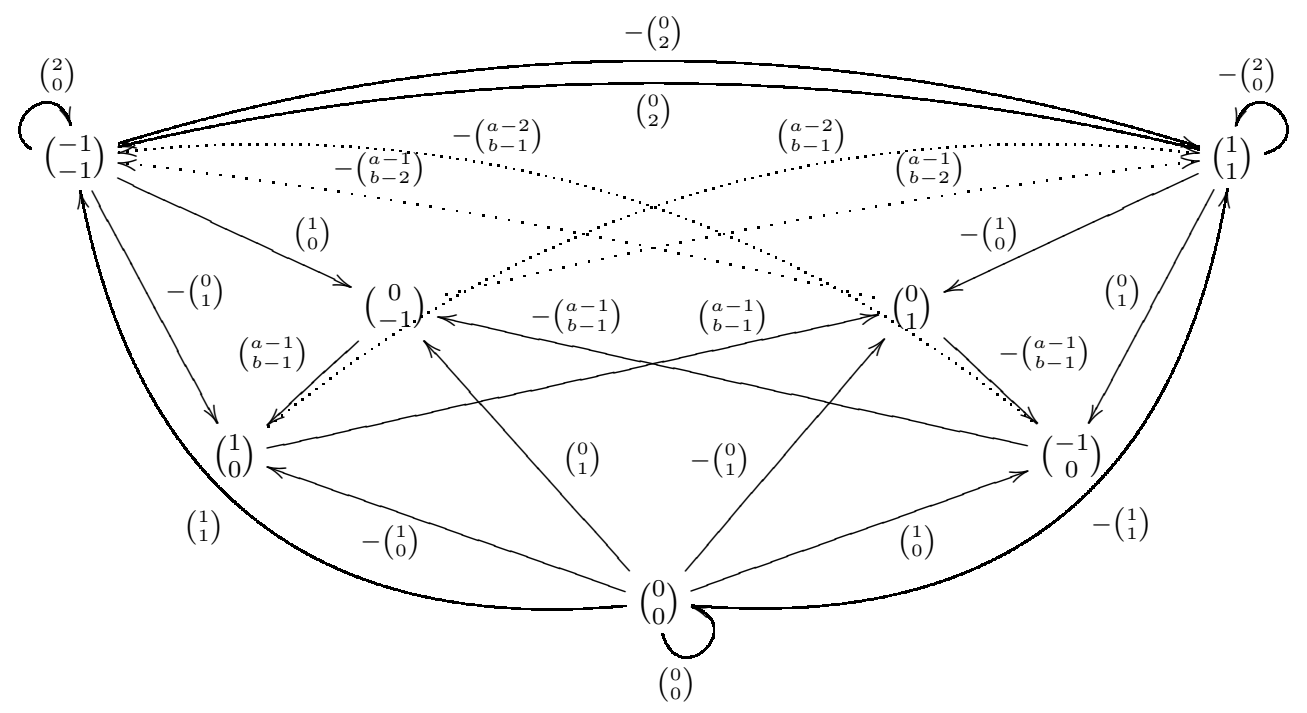

FIG. 9. Fifth graph

that are not removed until now:

$$
\begin{gathered}
(2,2) \stackrel{-\left(\begin{array}{l}
4 \\
0
\end{array}\right)}{\longrightarrow}(2,2),(1,2) \stackrel{-\left(\begin{array}{c}
a-1 \\
b-3
\end{array}\right)}{\longrightarrow}-(2,1),(1,2) \stackrel{-\left(\begin{array}{c}
a-1 \\
b-4
\end{array}\right)}{\longrightarrow}-(2,2),(2,1) \stackrel{\left(\begin{array}{c}
a-4 \\
b-1
\end{array}\right)}{\longrightarrow}(2,2), \\
(2,2) \stackrel{\left(\begin{array}{l}
0 \\
4
\end{array}\right)}{\longrightarrow}(-2,-2),(2,1) \stackrel{\left(\begin{array}{c}
a-3 \\
b-1
\end{array}\right)}{\longrightarrow}(1,2),(2,2) \stackrel{-\left(\begin{array}{l}
3 \\
0
\end{array}\right)}{\longrightarrow}(1,2),(2,2) \stackrel{\left(\begin{array}{l}
0 \\
3
\end{array}\right)}{\longrightarrow}(-2,-1) .
\end{gathered}
$$

If $|G(S)|=6$ we know that we certainly get a disk-like tile (cf. Remark 5.4). In case of $|G(S)| \geq 10$ we deduce by Proposition 2.6 that the arising tile is not disklike. For the situation $|G(S)|=8$ we make use of Proposition 2.6 and Lemma 2.9 to decide if $\mathcal{T}\left(A_{9}, \mathcal{D}\right)$ is disk-like or not. We know that $G(R \backslash\{0\})$ is a subgraph of $G(S)$. So $\{ \pm(1,0), \pm(0,1), \pm(1,1)\} \subset S$. Suppose that $(2,2) \in S$ and $|G(S)|=8$, i.e., $S=\{ \pm(1,0), \pm(0,1), \pm(1,1), \pm(2,2)\}$. Then $\mathcal{T}\left(A_{9}, \mathcal{D}\right)$ is not disk-like as the set $S$ cannot be written in the form $S=\{ \pm \alpha, \pm \beta, \pm(\alpha+\beta), \pm(\alpha-\beta)\}$ (cf. Proposition 2.6). The other two possibilities for $|G(S)|=8$, i.e.,

$$
\{ \pm \alpha, \pm \beta, \pm(\alpha+\beta), \pm(\alpha-\beta)\}=\{ \pm(1,0), \pm(0,1), \pm(1,1), \pm(1,2)\}
$$

and

$$
\{ \pm \alpha, \pm \beta, \pm(\alpha+\beta), \pm(\alpha-\beta)\}=\{ \pm(1,0), \pm(0,1), \pm(1,1), \pm(2,1)\}
$$

do not occur in this subsection as the product-graph $B[1] \otimes B[1]$ does not contain cycles of the form $(2,1) \rightarrow(2,1),(2,1) \rightarrow-(2,1) \rightarrow(2,1),(1,2) \rightarrow(1,2)$ and $(1,2) \rightarrow-(1,2) \rightarrow(1,2)$. Here we recall the fact that $G(S \cup\{0\})$ is the union of all cycles and paths connecting two cycles in $G\left(\mathbb{Z}^{2}\right)$.

We specify which sets $\mathcal{D}$ induce tiles that are disk-like. The following four conditions (A) to (D) are necessary for disk-likeness of the associated $\mathbb{Z}^{2}$-tile $\mathcal{T}$.

(A) We require $\left(\begin{array}{l}4 \\ 0\end{array}\right),\left(\begin{array}{l}0 \\ 4\end{array}\right) \notin \mathcal{D}-\mathcal{D}$ so that $(2,2)$ is not an element of $G(S)$. Otherwise we had a cycle $(2,2) \rightarrow(2,2)$ or $(2,2) \rightarrow-(2,2) \rightarrow(2,2)$, respectively. 
(B) If there is an edge $(1,2) \stackrel{-\left(\begin{array}{c}a-1 \\ b-3\end{array}\right)}{\longrightarrow}(-2,-1)$, we must not have the edge $(2,1) \stackrel{\left(\begin{array}{l}a-3 \\ b-1\end{array}\right)}{\longrightarrow}$ $(1,2)$ and vice versa. (Otherwise we had the cycle $(1,2) \rightarrow(-2,-1) \rightarrow(-1,-2) \rightarrow$ $(2,1) \rightarrow(1,2)$ and $|G(S)| \geq 10$. $)$ Thus $\left\{\left(\begin{array}{c}a-1 \\ b-3\end{array}\right),\left(\begin{array}{c}a-3 \\ b-1\end{array}\right)\right\} \not \subset \mathcal{D}-\mathcal{D}$.

(C) The condition $\left\{\left(\begin{array}{l}a-1 \\ b-4\end{array}\right),\left(\begin{array}{l}3 \\ 0\end{array}\right)\right\} \not \subset \mathcal{D}-\mathcal{D}$ assures that the cycles

$$
\pm(2,2) \rightarrow \pm(1,2) \rightarrow \mp(2,2) \rightarrow \mp(1,2) \rightarrow \pm(2,2)
$$

do not occur.

(D) Finally we require $\left\{\left(\begin{array}{l}a-4 \\ b-1\end{array}\right),\left(\begin{array}{l}0 \\ 3\end{array}\right)\right\} \not \subset \mathcal{D}-\mathcal{D}$ in order to avoid the cycles

$$
\pm(2,1) \rightarrow \pm(2,2) \rightarrow \mp(2,1) \rightarrow \mp(2,2) \rightarrow \pm(2,1) .
$$

On the other hand, if (A), (B), (C) and (D) holds the vertices $\pm(1,2), \pm(2,1)$ and $\pm(2,2)$ can be removed. Indeed, by inspecting (5.1) we see that (A) - (D) imply that those vertices are not a starting point of an infinite walk in $B[2]$.

In this case $B[2]=\operatorname{Red}(B[1] \otimes B[1])=B[1]$ and $\mathcal{T}$ is disk-like with hexagonal surrounding because $G(S)=B[1] \backslash\{0\}$ has $|S|=6$.

Summing up we get the following characterization: The tile $\mathcal{T}=\mathcal{T}\left(A_{9}, \mathcal{D}\right)$ is disk-like if and only if $\mathcal{D}-\mathcal{D}$ meets the conditions (A)-(D). In this case $\mathcal{T}$ has a hexagonal surrounding.

5.6. Sixth type of matrix. The graph $G(R)$ stemming from $\mathcal{T}\left(A_{10}, \mathcal{D}\right)$ with matrix $A_{10}=\left(\begin{array}{cc}a & -(a-1) \\ b-1 & -b\end{array}\right)$ is shown in Figure 10. This is the most involved case. We are going to compute the graph $G(S)$ by the help of Algorithm 2.4. The

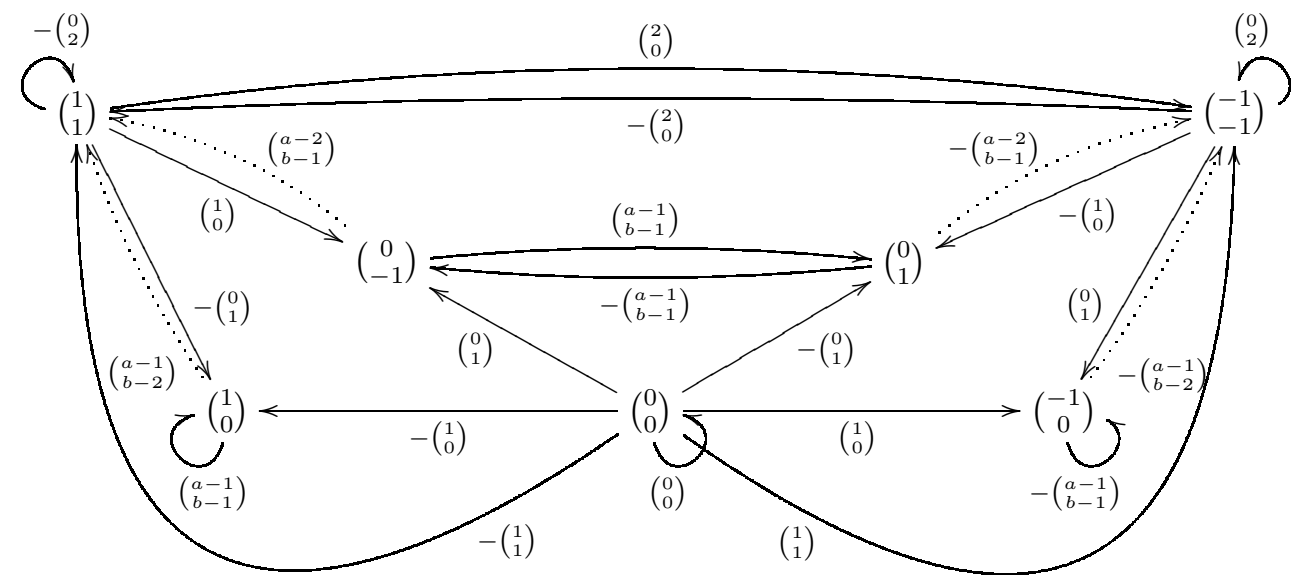

FIG. 10. Sixth graph

following states of the product-graph $B[1] \otimes B[1]$ do not belong to $G(R): \pm(2,0)$, $\pm(0,2), \pm(1,-1), \pm(2,1), \pm(1,2), \pm(2,2)$.

We start the reduction process and remove all states that are not starting points of paths of infinite length, i.e., $\pm(2,0), \pm(0,2)$, as the labels $\left(\begin{array}{c}2 a-2 \\ 2 b-2\end{array}\right),\left(\begin{array}{c}2 a-2 \\ 2 b-3\end{array}\right),\left(\begin{array}{c}2 a-2 \\ 2 b-4\end{array}\right)$, $\left(\begin{array}{l}2 a-3 \\ 2 b-2\end{array}\right)$ and $\left(\begin{array}{c}2 a-4 \\ 2 b-2\end{array}\right)$ are not elements of $\mathcal{D}-\mathcal{D}$. 
The only edge that leaves $(1,-1)$ is $(1,-1) \stackrel{\left(\begin{array}{l}2 a-3 \\ 2 b-3\end{array}\right)}{\longrightarrow}(2,2)$, provided that $a=b=2$ holds. As we are going to exclude $(2,2)$ from belonging to $G(S)$, we may remove $\pm(1,-1)$.

Now we write down all edges that leave the vertices $(2,1),(1,2),(2,2)$ :

$$
\begin{gathered}
(2,2) \stackrel{\left(\begin{array}{l}
4 \\
0
\end{array}\right)}{\longrightarrow}(-2,-2),(2,1) \stackrel{\left(\begin{array}{c}
a-1 \\
b-4
\end{array}\right)}{\longrightarrow}(2,2),(2,1) \stackrel{\left(\begin{array}{l}
a-1 \\
b-3
\end{array}\right)}{\longrightarrow}(2,1),(1,2) \stackrel{-\left(\begin{array}{l}
a-3 \\
b-1
\end{array}\right)}{\longrightarrow}(-1,-2), \\
(2,2) \stackrel{-\left(\begin{array}{l}
0 \\
4
\end{array}\right)}{\longrightarrow}(2,2),(1,2) \stackrel{-\left(\begin{array}{c}
a-4 \\
b-1
\end{array}\right)}{\longrightarrow}(-2,-2),(2,2) \stackrel{-\left(\begin{array}{l}
0 \\
3
\end{array}\right)}{\longrightarrow}(2,1),(2,2) \stackrel{\left(\begin{array}{l}
3 \\
0
\end{array}\right)}{\longrightarrow}(-1,-2) .
\end{gathered}
$$

We remember the remarks of the previous subsection: If $|G(S)|=6$ then $\mathcal{T}=$ $\mathcal{T}\left(A_{10}, \mathcal{D}\right)$ is disk-like and for $|G(S)| \geq 10$ it is certainly not. In case of $|G(S)|=8$ the situation is quite involved:

Suppose $\pm(2,2) \in G(S)$ then $\mathcal{T}$ is not disk-like by virtue of Proposition 2.6 and Lemma 2.9. Note that it is not possible to choose $\alpha$ and $\beta$ such that

$$
\{ \pm \alpha, \pm \beta, \pm(\alpha+\beta), \pm(\alpha-\beta)\}=\{ \pm(1,0), \pm(0,1), \pm(1,1), \pm(2,2)\}
$$

Now assume that $S=\{ \pm(1,0), \pm(0,1), \pm(1,1), \pm(2,1)\}$. We set $\alpha=(1,1), \beta=(1,0)$ in Lemma 2.9 and conclude that $\mathcal{D}$ is $\{ \pm \alpha, \pm \beta\}$-connected if and only if $\left(\begin{array}{l}0 \\ 2\end{array}\right) \notin \mathcal{D}-\mathcal{D}$. If this applies to $\mathcal{D}-\mathcal{D}$ then $\mathcal{T}\left(A_{10}, \mathcal{D}\right)$ is disk-like with parallelogramic surrounding. If $S=\{ \pm(1,0), \pm(0,1), \pm(1,1), \pm(1,2)\}$ we set $\alpha=(1,1), \beta=(0,1)$ in Lemma 2.9 . The set $\mathcal{D}$ is $\{ \pm \alpha, \pm \beta\}$-connected if and only if $\left(\begin{array}{l}2 \\ 0\end{array}\right) \notin \mathcal{D}-\mathcal{D}$. If this is fulfilled we get a disk-like tile $\mathcal{T}$ with parallelogramic surrounding.

Now we assure these results and give necessary and sufficient conditions for $\mathcal{D}$ such that $\mathcal{T}\left(A_{10}, \mathcal{D}\right)$ is disk-like. We are going to use the notation from Algorithm 2.4. Like in Subsection 5.5 we start with four necessary conditions (A) to (D) for disk-likeness of $\mathcal{T}$.

(A) We require $\left(\begin{array}{l}4 \\ 0\end{array}\right),\left(\begin{array}{l}0 \\ 4\end{array}\right) \notin \mathcal{D}-\mathcal{D}$, as otherwise $\pm(2,2) \in B[2]=\operatorname{Red}(B[1] \otimes B[1])$.

(B) The condition $\left\{\left(\begin{array}{c}a-1 \\ b-3\end{array}\right),\left(\begin{array}{l}0 \\ 3\end{array}\right)\right\} \not \subset \mathcal{D}-\mathcal{D}$ is necessary to avoid $\{ \pm(2,2), \pm(2,1)\} \subset B[2]$.

(C) The condition $\left\{\left(\begin{array}{l}a-3 \\ b-1\end{array}\right),\left(\begin{array}{l}3 \\ 0\end{array}\right)\right\} \not \subset \mathcal{D}-\mathcal{D}$ is necessary to avoid $\{ \pm(2,2), \pm(1,2)\} \subset B[2]$.

(D) The condition $\left\{\left(\begin{array}{c}a-3 \\ b-1\end{array}\right),\left(\begin{array}{c}a-1 \\ b-3\end{array}\right)\right\} \not \subset \mathcal{D}-\mathcal{D}$ is necessary to avoid $\{ \pm(2,1), \pm(1,2)\} \subset$ $B[2]$.

If the set $\mathcal{D}-\mathcal{D}$ meets the conditions $(\mathrm{A})-(\mathrm{D})$, then in view of (5.2) we either have $|B[2]|=7$ or $|B[2]|=9$. In the first case Algorithm 2.4 terminates, we get $|G(S)|=6$ and the tile $\mathcal{T}$ is disk-like, cf. Remark 5.4. We have to take a closer look at the possibility $|B[2]|=9$ which is done in $(\mathrm{E})$ and $(\mathrm{F})$.

(E) If $B[2]$ has the vertices $\{(0,0), \pm(1,0), \pm(0,1), \pm(1,1), \pm(2,1)\}$, then a necessary (and sufficient) condition for the disk-likeness of $\mathcal{T}\left(A_{10}, \mathcal{D}\right)$ is $B[3]=B[2]$ and $\left(\begin{array}{l}0 \\ 2\end{array}\right) \notin$ $\mathcal{D}-\mathcal{D}$ so that we have $S=\{ \pm(1,0), \pm(0,1), \pm(1,1), \pm(2,1)\}$. We have already explained in detail why this gives a disk-like tile. Keep in mind that in this case the label $\left(\begin{array}{c}a-1 \\ b-3\end{array}\right)$ must be an element of $\mathcal{D}-\mathcal{D}$. Otherwise the cycle $(2,1) \rightarrow(2,1)$ would not exist and as a consequence $\pm(2,1)$ were not a vertex of $B[2]$ contrary to our assumption.

We are going to apply Algorithm 2.4 and verify $B[3]=B[2]$. The states of $B[2] \otimes B[1]$ which are not elements of $B[2]$ are $\pm(3,1), \pm(2,2), \pm(3,2)$ and $\pm(2,0)$. We check if there are edges leaving the vertex $(3,1)$ in $B[2] \otimes B[1]$. The only candidates are $(3,1) \stackrel{\left(\begin{array}{l}2 a-2 \\ 2 b-4\end{array}\right)}{\longrightarrow}(3,1)$ and $(3,1) \stackrel{\left(\begin{array}{l}2 a-2 \\ 2 b-5\end{array}\right)}{\longrightarrow}(3,2)$. The labels $\left(\begin{array}{c}2 a-2 \\ 2 b-4\end{array}\right)$ and $\left(\begin{array}{l}2 a-2 \\ 2 b-5\end{array}\right)$ are not elements of $\mathcal{D}-\mathcal{D}$ and so there are no edges leaving $(3,1)$. We remove $\pm(3,1)$. The 
states $\pm(2,2)$ are also deleted as the edges $(2,2) \stackrel{-\left(\begin{array}{l}0 \\ 2\end{array}\right)}{\longrightarrow}(2,0),(2,2) \stackrel{\left(\begin{array}{c}1 \\ -2\end{array}\right)}{\longrightarrow}(1,0)$ are not feasible due to $\left(\begin{array}{c}0 \\ 2\end{array}\right),\left(\begin{array}{c}1 \\ -2\end{array}\right) \notin \mathcal{D}-\mathcal{D}$. Now we investigate if there are edges leaving $(3,2)$. We have to check $(3,2) \stackrel{\left(\begin{array}{l}a-1 \\ b-5\end{array}\right)}{\longrightarrow}(3,2),(3,2) \stackrel{\left(\begin{array}{l}a+1 \\ b-3\end{array}\right)}{\longrightarrow}(1,0),(3,2) \stackrel{\left(\begin{array}{c}a \\ b-3\end{array}\right)}{\longrightarrow}(2,0)$ and $(3,2) \stackrel{\left(\begin{array}{l}a-1 \\ b-4\end{array}\right)}{\longrightarrow}(3,1)$. It turns out that none of the labels is an element of $\mathcal{D}-\mathcal{D}$. For $\left(\begin{array}{l}a+1 \\ b-3\end{array}\right)$ and $\left(\begin{array}{c}a \\ b-3\end{array}\right)$ this is easily seen, whereas $\left(\begin{array}{c}a-1 \\ b-5\end{array}\right),\left(\begin{array}{c}a-1 \\ b-4\end{array}\right) \notin \mathcal{D}-\mathcal{D}$ is a direct consequence of $\left(\begin{array}{l}0 \\ 2\end{array}\right) \notin \mathcal{D}-\mathcal{D}$. This is why we remove $\pm(3,2)$. Finally we focus on $(2,0)$. The possibilities $(2,0) \stackrel{\left(\begin{array}{l}2 a-3 \\ 2 b-4\end{array}\right)}{\longrightarrow}(3,2)$ and $(2,0) \stackrel{\left(\begin{array}{l}2 a-2 \\ 2 b-4\end{array}\right)}{\longrightarrow}(2,2)$ drop out as the states $(3,2)$ and $(2,2)$ are already removed. This means that $B[3]=B[2]$ holds and we are done.

Recapitulating we can make the following note: Assume that $\left(\begin{array}{l}a-1 \\ b-3\end{array}\right) \in \mathcal{D}-\mathcal{D}$ holds. Then the condition $\left(\begin{array}{l}4 \\ 0\end{array}\right),\left(\begin{array}{l}0 \\ 2\end{array}\right) \notin \mathcal{D}-\mathcal{D}$ is necessary and sufficient for the tile $\mathcal{T}\left(A_{10}, \mathcal{D}\right)$ to be disk-like. This immediately follows from $(\mathrm{A})-(\mathrm{D})$ and the additional condition $\left(\begin{array}{l}0 \\ 2\end{array}\right) \notin \mathcal{D}-\mathcal{D}$ based upon Lemma 2.9.

(F) Let $S=\{ \pm(1,0), \pm(0,1), \pm(1,1), \pm(1,2)\}$, then $\left(\begin{array}{l}2 \\ 0\end{array}\right) \notin \mathcal{D}-\mathcal{D}$ is necessary so that $\mathcal{D}$ is $\{ \pm(1,1), \pm(0,1)\}$-connected. It turns out that this case bears analogy to (E). Here we start with $\left(\begin{array}{l}a-3 \\ b-1\end{array}\right) \in \mathcal{D}-\mathcal{D}$ and the condition $\left(\begin{array}{l}2 \\ 0\end{array}\right) \notin \mathcal{D}-\mathcal{D}$. Again we apply Algorithm 2.4 and show that $B[3]=B[2]$ holds. Then it is easy to formulate the following result:

Suppose that $\left(\begin{array}{l}a-3 \\ b-1\end{array}\right) \in \mathcal{D}-\mathcal{D}$ holds. Then the condition $\left(\begin{array}{l}0 \\ 4\end{array}\right),\left(\begin{array}{l}2 \\ 0\end{array}\right) \notin \mathcal{D}-\mathcal{D}$ is necessary and sufficient for the tile $\mathcal{T}\left(A_{10}, \mathcal{D}\right)$ to be disk-like.

Altogether we get the following result: The tile $\mathcal{T}\left(A_{10}, \mathcal{D}\right)$ is disk-like if and only if the following conditions are fulfilled:

- $\left(\begin{array}{l}4 \\ 0\end{array}\right),\left(\begin{array}{l}0 \\ 4\end{array}\right) \notin \mathcal{D}-\mathcal{D}$,

- $\left(\begin{array}{c}a-1 \\ b-3\end{array}\right) \in \mathcal{D}-\mathcal{D} \Rightarrow\left(\begin{array}{l}0 \\ 2\end{array}\right) \notin \mathcal{D}-\mathcal{D}$,

- $\left(\begin{array}{c}a-3 \\ b-1\end{array}\right) \in \mathcal{D}-\mathcal{D} \Rightarrow\left(\begin{array}{l}2 \\ 0\end{array}\right) \notin \mathcal{D}-\mathcal{D}$.

6. Examples. We finish this paper with some examples. Consider the tiles $\mathcal{T}_{1}$, $\mathcal{T}_{2}$ and $\mathcal{T}_{3}$ corresponding to the matrix $A_{10}$ with $a=b=4$ and the digit sets given in Figure 1 , respectively. For $\mathcal{T}_{1}$ we see that $\left(\begin{array}{l}4 \\ 0\end{array}\right)$ and $\left(\begin{array}{l}0 \\ 4\end{array}\right)$ are not in $\mathcal{D}-\mathcal{D}$. The same

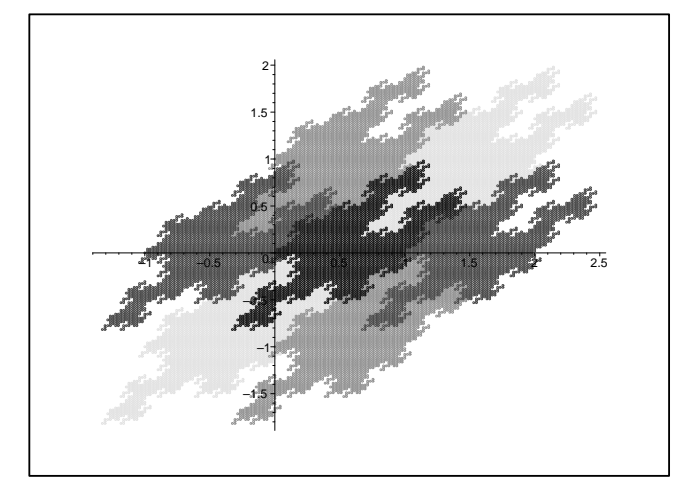

FIG. 11. The tile $\mathcal{T}_{1}$ with its hexagonal surrounding

holds for $\left(\begin{array}{l}a-1 \\ b-3\end{array}\right)=\left(\begin{array}{l}3 \\ 1\end{array}\right)$ and $\left(\begin{array}{l}a-3 \\ b-1\end{array}\right)=\left(\begin{array}{l}1 \\ 3\end{array}\right)$ thus the tile is disk-like. From the proof in Section 5.6 we even see that it has 6 neighbors, i.e., a hexagonal surrounding. The tile $\mathcal{T}_{1}$ is depicted in Figure 11. 
Similarly, one can see that $\mathcal{T}_{2}$, which is depicted in Figures 12 is disk-like. In fact,

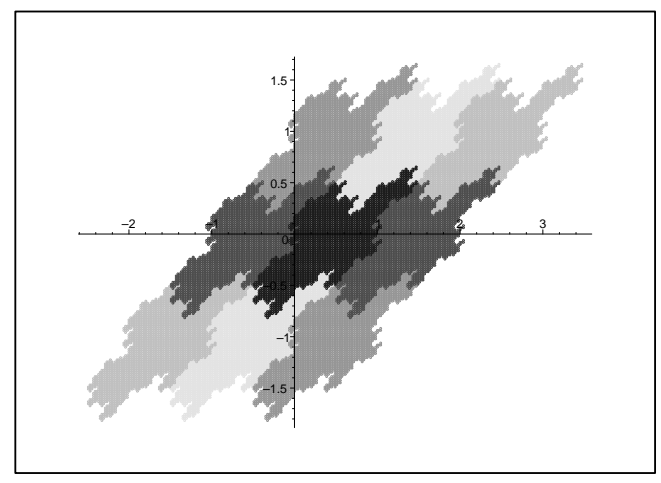

FIG. 12. The tile $\mathcal{T}_{2}$ with its parallelogramic surrounding

again $\left(\begin{array}{l}4 \\ 0\end{array}\right),\left(\begin{array}{l}0 \\ 4\end{array}\right)$ and $\left(\begin{array}{l}a-3 \\ b-1\end{array}\right)=\left(\begin{array}{l}1 \\ 3\end{array}\right)$ are not in $\mathcal{D}-\mathcal{D}$. However, $\left(\begin{array}{l}a-1 \\ b-3\end{array}\right)=\left(\begin{array}{l}3 \\ 1\end{array}\right) \in \mathcal{D}-\mathcal{D}$. But since $\left(\begin{array}{l}0 \\ 2\end{array}\right) \notin \mathcal{D}-\mathcal{D}$ disk-likeness follows. Again by inspecting the proof in Section 5.6 we see that $\mathcal{T}_{2}$ has a parallelogramic surrounding with 4 edge neighbors and 4 vertex neighbors.

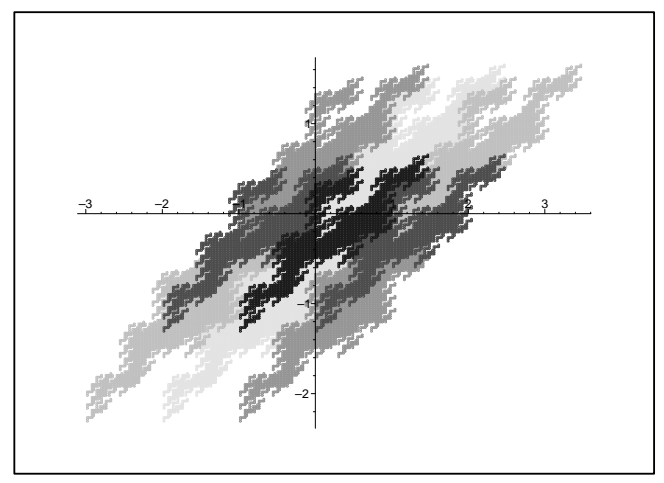

FIG. 13. The tile $\mathcal{T}_{3}$ is not disk-like and has 8 neighbors

Finally, for $\mathcal{T}_{3}$ we see that $\left(\begin{array}{l}a-1 \\ b-3\end{array}\right)=\left(\begin{array}{l}3 \\ 1\end{array}\right) \in \mathcal{D}-\mathcal{D}$. But since also $\left(\begin{array}{l}0 \\ 2\end{array}\right) \in \mathcal{D}$ we conclude that $\mathcal{T}_{3}$ is not disk-like. It has 8 neighbors. Together with these neighbors it is depicted in Figure 13.

Acknowledgement. The authors are very grateful to the anonymous referee for many valuable comments that really helped them in order to improve the paper.

\section{REFERENCES}

[1] Akiyama, S., And Guni, N., On the connectedness of self-affine attractors, Arch. Math. (Basel), 82 (2004), pp. 153-163.

[2] AkiYama, S., And Thuswaldner, J. M., On the topological structure of fractal tilings generated by quadratic number systems, Comp. Math. Appl., 49 (2005), pp. 1439-1485.

[3] Akiyama, S., and Thuswaldner, J. M., A survey on topological properties of tiles related to number systems, Geom. Dedicata, 109 (2004), pp. 89-105.

[4] Bandt, C., And Wang, Y., Disk-like self-affine tiles in $\mathbb{R}^{2}$, Discrete Comput. Geom., 26 (2001), pp. $591-601$. 
[5] Beauquier, D., And Nivat, M., On translating one polyomino to tile the plane, Discrete Comput. Geom., 6 (1991), pp. 575-592.

[6] Golomb, S. W., Polyominoes: puzzles, patterns, problems, Princeton University Press, Princeton, NJ, 1995.

[7] Gröchenig, K., and HaAs, A., Self-similar lattice tilings, J. Fourier Anal. Appl., 1 (1994), pp. $131-170$.

[8] Hata, M., On the structure of self-similar sets, Japan J. Appl. Math., 2 (1985), pp. 381-414.

[9] Hata, M., Topological aspects of self-similar sets and singular functions, in Fractal geometry and analysis (Montreal, PQ, 1989), vol. 346 of NATO Adv. Sci. Inst. Ser. C Math. Phys. Sci., Kluwer Acad. Publ., Dordrecht, 1991, pp. 255-276.

[10] Hutchinson, J. E., Fractals and self-similarity, Indiana Univ. Math. J., 30 (1981), pp. 713-747.

[11] Keating, K., And Vince, A., Isohedral polyomino tiling of the plane, Discrete Comput. Geom., 21 (1999), pp. 615-630.

[12] Kirat, I., And LaU, K.-S., On the connectedness of self-affine tiles, J. London Math. Soc. (2), 62 (2000), pp. 291-304.

[13] Kirat, I., LAU, K.-S., AND RaO, H., Expanding polynomials and connectedness of self-affine tiles, Discrete Comput. Geom., 31 (2004), pp. 275-286.

[14] Lagarias, J., AND WANG, Y., Integral self-affine tiles in $\mathbb{R}^{n}$ I. standard and nonstandard digit sets, J. London Math. Soc., 54 (1996), pp. 161-179.

[15] Lagarias, J., And Wang, Y., Self-affine tiles in $\mathbb{R}^{n}$, Adv. Math., 121 (1996), pp. 21-49.

[16] Luo, J., RAO, H., AND TAN, B., Topological structure of self-similar sets, Fractals, 10 (2002), pp. 223-227.

[17] Müller, W., Thuswaldner, J. M., And Tichy, R. F., Fractal properties of number systems, Period. Math. Hungar., 42 (2001), pp. 51-68.

[18] NGai, S.-M., AND T.-M., TANG, Topology of connected self-similar tiles in the plane with disconnected interiors, Topology Appl., 150 (2005), pp. 139-155.

[19] NGaI, S.-M., AND T.-M., TANG, Vertices of self-similar tiles, Illinois J. Math., 49 (2005), pp. $857-872$.

[20] NGaI, S.-M., And T.-M., TAnG, A technique in the topology of connected self-similar tiles, Fractals, 12 (2004), pp. $389-403$.

[21] Scheicher, K., And Thuswaldner, J. M., Neighbours of self-affine tiles in lattice tilings, in Fractals in Graz 2001, Trends Math., Birkhäuser, Basel, 2003, pp. 241-262.

[22] Song, H. J., And Kang, B. S., Disclike lattice reptiles induced by exact polyominoes, Fractals, 7 (1999), pp. 9-22.

[23] Strichartz, R. S., And Wang, Y., Geometry of self-affine tiles. I, Indiana Univ. Math. J., 48 (1999), pp. 1-23.

[24] Vince, A., Digit tiling of Euclidean space, in Directions in mathematical quasicrystals, Amer. Math. Soc., Providence, RI, 2000, pp. 329-370. 
